\title{
Measurement of CP Violation in Stop Cascade Decays at the LHC
}

\section{Journal Article}

Author(s):

Ellis, John; Moortgat, Filip; Moortgat-Pick, Gudrid; Smillie, Jennifer M.; Tattersall, Jamie

Publication date:

2009-04

Permanent link:

https://doi.org/10.3929/ethz-b-000011942

Rights / license:

Creative Commons Attribution 4.0 International

Originally published in:

The European Physical Journal C 60(4), https://doi.org/10.1140/epjc/s10052-009-0964-8 


\title{
Measurement of CP violation in stop cascade decays at the LHC
}

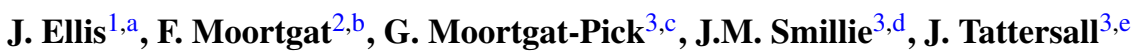 \\ ${ }^{1}$ Theory Division, Physics Department, CERN, 1211 Geneva 23, Switzerland \\ ${ }^{2}$ Department of Physics, ETH Honggerberg, 8093 Zurich, Switzerland \\ ${ }^{3}$ IPPP, University of Durham, Durham DH1 3LE, UK
}

Received: 10 October 2008 / Revised: 20 October 2008 / Published online: 12 March 2009

(C) Springer-Verlag / Società Italiana di Fisica 2009

\begin{abstract}
We study the potential observation at the LHC of $\mathrm{CP}$-violating effects in stop production and subsequent cascade decays, $g g \rightarrow \tilde{t}_{i} \tilde{t}_{i}, \tilde{t}_{i} \rightarrow t \tilde{\chi}_{j}^{0}, \tilde{\chi}_{j}^{0} \rightarrow \tilde{\chi}_{1}^{0} \ell^{+} \ell^{-}$, within the Minimal Supersymmetric Standard Model. We study Todd asymmetries based on triple products between the different decay products. There may be a large CP asymmetry at the parton level, but there is a significant dilution at the hadronic level after integrating over the parton distribution functions. Consequently, even for scenarios where large CP intrinsic asymmetries are expected, the measurable asymmetry is rather small. High luminosity and precise measurements of masses, branching ratios and $\mathrm{CP}$ asymmetries may enable measurements of the $\mathrm{CP}$-violating parameters in cascade decays at the LHC.
\end{abstract}

\section{Introduction}

The Minimal Supersymmetric Standard Model (MSSM) is a particularly compelling extension of the Standard Model that may soon be explored at the Large Hadron Collider (LHC). Current data suggest that, if the MSSM is realised in Nature, the supersymmetry scale should easily be within reach of the LHC design centre-of-mass energy of $14 \mathrm{TeV}[1,2]$. If supersymmetry is discovered, many studies will be required to determine the exact details of its realisation.

The MSSM contains a large number of (as yet) undetermined parameters that may have non-zero phases $[3,4]$. Many of these phases are unphysical in the sense that they can be absorbed into the definitions of the fields; however,

\footnotetext{
a e-mail: John.Ellis@cern.ch

b e-mail: filip.moortgat@cern.ch

c e-mail: g.a.moortgat-pick@durham.ac.uk

de-mail: j.m.smillie@durham.ac.uk

e e-mail: jamie.tattersall@durham.ac.uk
}

not all phases can be consistently removed in this way. In the neutralino/chargino sector of the complex MSSM, the phase of the $\mathrm{SU}(2)$ gaugino mass $M_{2}$ is usually absorbed, whereas the phases of the $\mathrm{U}(1)$ gaugino mass $M_{1}$ and the Higgsino mixing parameter $\mu$ are generally left manifest; this is the parameterisation we use. The trilinear couplings $A_{f}$ can also be complex. Studies of these CP-violating parameters in sparticle decays and via other properties measurable at the LHC will be challenging [5, 6]. However, they are extremely important and provide a valuable training ground for exploring the limits of the LHC's capabilities.

Certain combinations of the CP-violating MSSM phases are constrained by the experimental upper bounds on the electric dipole moments (EDMs) of the electron, neutron and atoms, notably ${ }^{205} \mathrm{Tl}$ and ${ }^{199} \mathrm{Hg}$. Ignoring possible cancellations, the most severely constrained individual phase in the MSSM is that of $\mu$, which contributes at the one-loop level. For $O(100) \mathrm{GeV}$ masses, one must require $\left|\phi_{\mu}\right| \lesssim 0.1$. However, this restriction can be relaxed if the masses of the first- and second-generation squarks are large $(>\mathrm{TeV})$ while the third-generation masses remain relatively small $(<\mathrm{TeV})$, or in the presence of cancellations between the contributions of different CP-violating phases. We note that $M_{1}$ also contributes at the one-loop level, but again, if accidental cancellations are allowed between terms, it remains essentially unconstrained. The phases of the third-generation trilinear couplings, $\phi_{A_{t, b, \tau}}$ have weaker constraints, as they contribute to EDMs only at the two-loop level. Again, accidental cancellations can occur that weaken further the constraints: see [7-17]. A comprehensive summary of the EDM constraints and other CP-violating effects in SUSY is given in [18]. Here we study the complete range of $\mathrm{CP}$ phases in order to see the general dependences exhibited by our observables, and what luminosity might be required to observe these within the LHC environment. Therefore, we do not calculate explicitly which values of the other phases might be required for the points in our displayed scenarios to satisfy the EDM constraints. 
The precise determination of these phases is expected to be possible only at an $e^{+} e^{-}$linear collider, for instance at the planned International Linear Collider (ILC) or at the Compact Linear Collider (CLIC). However, it will be crucial for such future search strategies to use LHC data to learn as much as possible, as early as possible. Furthermore, the combination of independent measurements at the LHC and a linear collider will be important to determine the underlying model.

In this paper we concentrate on the potential for observing unique CP-violating effects in decay chains at the LHC, and investigate the circumstances under which a determination of the complex MSSM phases may be achievable, possibly with the support of other LHC measurements.

Specifically, we consider the LHC process $g g \rightarrow \tilde{t} \overline{\tilde{t}}$, with subsequent decay $\tilde{t} \rightarrow t \tilde{\chi}_{2}^{0}, \tilde{\chi}_{2}^{0} \rightarrow \ell^{+} \ell^{-} \tilde{\chi}_{1}^{0}$. We consider the situation where the $\tilde{\chi}_{2}^{0}$ decay is a three-body decay; this leads to $\mathrm{CP}$ violation as there is a non-negligible contribution from interference diagrams. This process involves the three phases $\phi_{M_{1}}, \phi_{\mu}$ and $\phi_{A_{t}}$; we discuss below the combinations to which this process is sensitive. We extract information on the phases using triple products formed from the decay products of the stop. Such T-odd variables have also been studied in the context of heavy squark and stau decays in [6, 19-23]. Other related studies are [24-34].

The first CP-odd asymmetry we consider is formed from $\mathcal{T}_{t}=\mathbf{p}_{t} \cdot\left(\mathbf{p}_{\ell^{+}} \times \mathbf{p}_{\ell^{-}}\right)$. This quantity has been studied at the parton level in [6], assuming pure gaugino-like neutralinos. In our current study we provide analytic expressions for the squared amplitude of the cascade process including full spin correlations and general neutralino mixing, and also provide an analytic expression for the phase space in the laboratory system. We also incorporate parton density functions (pdfs) and discuss the $\mathrm{CP}$-odd observables at both the parton and the hadronic levels. Transition to the latter level has a big dilution effect on the measurability of a CP-odd asymmetry. We include the possible LHC uncertainties in masses and asymmetries and discuss the extent to which CP-violating phases may be constrained in such cascade decays at the LHC.

In [21], further CP sensitive asymmetries formed from the momentum of the $b$ quark in the top decay were studied under the assumption of 2-body neutralino decays into on-shell sleptons, namely $\mathcal{T}_{b}=\mathbf{p}_{b} \cdot\left(\mathbf{p}_{\ell^{+}} \times \mathbf{p}_{\ell^{-}}\right)$and $\mathcal{T}_{t b}=$ $\mathbf{p}_{b} \cdot\left(\mathbf{p}_{t} \times \mathbf{p}_{\ell^{ \pm}}\right)$. These variables are sensitive to $\phi_{M_{1}}$ and $\phi_{A_{t}}$, but they have different dependences on the CP-violating phases as described in Sect. 2.3. Therefore, a combination of all three observables would in principle allow one to disentangle the influences of all three phases.

Since T-odd observables can also be generated by finalstate interactions at the one-loop level, one should in principle combine the asymmetry for a process with that one of its charge-conjugated process. If a non-zero asymmetry is then observed in this combination, it must correspond to a violation of CP symmetry. For the triple product, $\mathcal{T}_{b}$, this is experimentally possible as long as the associated $W$ decays into a final-state lepton, which enables us to determine the change of the $\tilde{t}$. Regarding the other triple products, we require information from the opposite decay chain to identify the charge. In all the scenarios we consider, the decay $\tilde{t} \rightarrow \tilde{\chi}_{i}^{+} b$, is dominant, enabling charge identification in principle. However, a detailed simulation including all combinatorial aspects and also other background processes would be required to validate this possibility.

We begin by describing the process under consideration in Sect. 2, including the phases involved and their various effects. In Sect. 3 we present numerical results for three specific benchmark scenarios and discuss the potential for a measurement at the LHC. The appendices contain details of the Lagrangian, the expression for the squared amplitude including full spin correlations, and the kinematics of the phase space in the laboratory system.

\section{Formalism}

2.1 The process studied and its squared amplitude

We study the dominant stop production process at the LHC, namely

$g g \rightarrow \tilde{t}_{i} \tilde{\bar{t}}_{i}$,

with the subsequent decay chain

$\tilde{t}_{i} \rightarrow \tilde{\chi}_{j}^{0}+t \rightarrow \tilde{\chi}_{1}^{0} \ell^{+} \ell^{-}+W b$.

At tree level, the production process (2.1) proceeds via $g$ exchange in the direct channel and $\tilde{t}$ exchange in the crossed channel, and via a quartic coupling, as shown in Fig. 2.1. Another possible source of $\tilde{t}_{1} \mathrm{~s}$ is their production in gluino decays, $\tilde{g} \rightarrow \tilde{t} t$. However this leads to an experimentally more complex topology than the direct production and
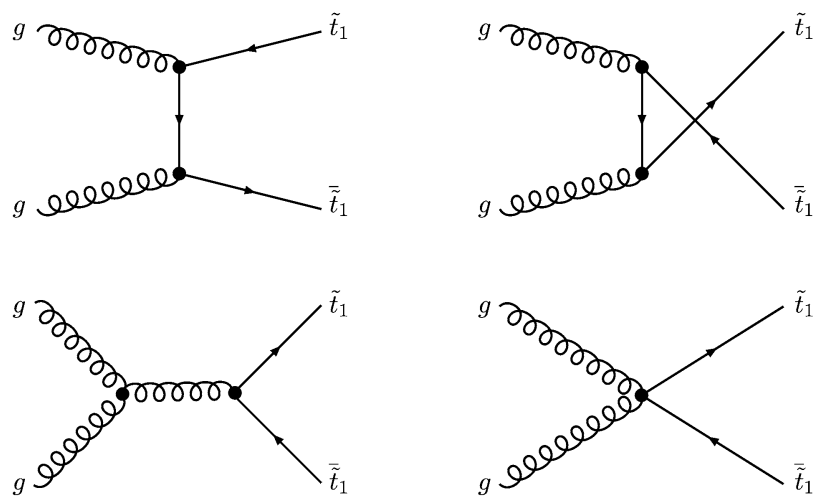

Fig. 2.1 Feynman diagrams for the production process $g g \rightarrow \tilde{t}_{1} \overline{\tilde{t}}_{1}$ 
consequently we do not investigate this channel. The Lagrangian and the resulting neutralino and stop mixings and couplings are described in Appendix A.

Since gluons do not couple to off-diagonal combinations of stop mass eigenstates of opposite chirality, and similarly for stop exchange and the quadratic couplings, $\tilde{t}_{1} \overline{\tilde{t}}_{2}$ production occurs only at the loop level, and we do not consider it here. We focus here on $\tilde{t}_{1} \overline{\tilde{t}}_{1}$ production, since the reconstruction of full decay chains of $\tilde{t}_{1}$ seems achievable, even in the complex experimental environment at the LHC. With the exception of the stop mass eigenvalues, see Appendix A, no effects from supersymmetric $\mathrm{CP}$-violating couplings occur in the tree-level production process.

The first step in the cascade decay chain is the two-body process $\tilde{t}_{i} \rightarrow t \tilde{\chi}_{2}^{0}$. Here CP-violating couplings of the $\tilde{t}_{1}$ enter as well as those of the $\tilde{\chi}_{2}^{0}$, and are dominated by the phases $\phi_{A_{t}}$ and $\phi_{M_{1}}$; see Appendix B.1. ${ }^{1}$ Since constraints from electric dipole measurements strongly constrain $\phi_{\mu}$, we set $\phi_{\mu}=0$ in our study [35-37]. We consider spectra where the second steps in the cascade decay chains are the three-body decays of the neutralino, $\tilde{\chi}_{2}^{0} \rightarrow \tilde{\chi}_{1}^{0} \ell^{+} \ell^{-}$(cf. Appendix B.2) and the dominant top decay $t \rightarrow W b$ (cf. Appendix B.3). The neutralino decay occurs via $Z^{0}$ exchange in the direct channel and via $\tilde{\ell}_{L, R}$ exchanges in the crossed channels, cf. Fig. 2.2. It is very sensitive to CP-violating supersymmetric couplings, and its structure has been studied in detail in $[38,39]$. The phase $\phi_{M_{1}}$ (and also $\phi_{\mu}$, which has been set to zero here) affects the mass of the $\tilde{\chi}_{2}^{0}$, as well as its couplings and decay rates.

Using the formalism of $[39,40]$, the squared amplitude $|T|^{2}$ of the full process can be factorised into the processes of production $g g \rightarrow \tilde{t}_{1} \overline{\tilde{t}}_{1}$ and the subsequent decays $\tilde{t}_{1} \rightarrow$ $t \tilde{\chi}_{2}^{0}, \tilde{\chi}_{2}^{0} \rightarrow \tilde{\chi}_{1}^{0} \ell^{+} \ell^{-}$and $t \rightarrow W b$, with the second $\tilde{t}_{1}$ being unobserved. We apply the narrow-width approximation for the masses of the intermediate particles, $\tilde{t}_{1}, \tilde{\chi}_{2}^{0}$ and $t$, which is appropriate since the widths of the respective particles are in all cases much smaller than their masses, cf. Table 3.1. the squared amplitude can then be expressed in the form

$$
\begin{aligned}
|T|^{2}= & 4\left|\Delta\left(\tilde{t}_{1}\right)\right|^{2}\left|\Delta\left(\tilde{\chi}_{2}^{0}\right)\right|^{2}|\Delta(t)|^{2} P\left(\tilde{t}_{1} \tilde{t}_{1}\right) \\
& \times\left\{P\left(\tilde{\chi}_{2}^{0} t\right) D\left(\tilde{\chi}_{2}^{0}\right) D(t)+\sum_{a=1}^{3} \Sigma_{P}^{a}\left(\tilde{\chi}_{2}^{0}\right) \Sigma_{D}^{a}\left(\tilde{\chi}_{2}^{0}\right) D(t)\right. \\
& +\sum_{b=1}^{3} \Sigma_{P}^{b}(t) \Sigma_{D}^{b}(t) D\left(\tilde{\chi}_{2}^{0}\right) \\
& \left.+\sum_{a, b=1}^{3} \Sigma_{P}^{a b}\left(\tilde{\chi}_{2}^{0} t\right) \Sigma_{D}^{a}\left(\tilde{\chi}_{2}^{0}\right) \Sigma_{D}^{b}(t)\right\}
\end{aligned}
$$

\footnotetext{
${ }^{1}$ Their structure has also been studied in detail in [21].
}

where $a=1,2,3$ refers to the polarisation states of the neutralino $\tilde{\chi}_{i}^{0}$ and top quark, which are described by the polarisation vectors $s^{a}\left(\tilde{\chi}_{i}^{0}\right), s^{b}(t)$ given in Appendix B.1. In addition, we have the following.

- $\Delta\left(\tilde{t}_{1}\right), \Delta\left(\tilde{\chi}_{2}^{0}\right)$ and $\Delta(t)$ are the 'propagators' of the intermediate particles which lead to the factors $E_{\tilde{t}_{1}} / m_{\tilde{t}_{1}} \Gamma_{\tilde{t}_{1}}$, $E_{\tilde{\chi}_{2}^{0}} / m_{\tilde{\chi}_{2}^{0}} \Gamma_{\tilde{\chi}_{2}^{0}}$ and $E_{t} / m_{t} \Gamma_{t}$ in the narrow-width approximation.

- $P\left(\tilde{t}_{1} \tilde{t}_{1}\right), P\left(t \tilde{\chi}_{2}^{0}\right), D\left(\tilde{\chi}_{i}^{0}\right)$ and $D(t)$ are the terms in the production and decay that are independent of the polarisations of the decaying neutralino and top, whereas

- $\Sigma_{P}^{a}\left(\tilde{\chi}_{i}^{0}\right), \Sigma_{P}^{b}(t), \Sigma_{P}^{a b}\left(\tilde{\chi}_{2}^{0} t\right)$ and $\Sigma_{D}^{a}\left(\tilde{\chi}_{i}^{0}\right), \Sigma_{D}^{b}(t)$ are the terms containing the correlations between production and decay spins of the $\tilde{\chi}_{2}^{0}$ and $t$.

According to our choice of the polarisation vectors $s^{a}\left(\tilde{\chi}_{i}^{0}\right)\left[s^{b}(t)\right]$, see (B.8)-(B.13) in Appendix B.1, $\Sigma_{P}^{3} / P$ is the longitudinal polarisation, $\Sigma_{P}^{1} / P$ is the transverse polarisation in the production plane, and $\Sigma_{P}^{2} / P$ is the polarisation perpendicular to the reference plane of the neutralino $\tilde{\chi}_{i}^{0}[$ top quark $t]$.

\subsection{Cross section for the whole process at parton level}

The differential cross section in the laboratory system is

$d \sigma=\frac{1}{8 E_{b}^{2}}|T|^{2}(2 \pi)^{4} \delta^{4}\left(p_{1}+p_{2}-\sum_{n} p_{n}\right) d \operatorname{lips}\left(p_{n}\right)$,

where $E_{b}$ is the beam energy of the gluons, $p_{1}$ and $p_{2}$ are the momenta of the incoming gluons, the $p_{n}$ are the momenta of the outgoing particles and $d \operatorname{lips}\left(p_{n}\right)$ is the Lorentzinvariant phase-space element. Integrating over all angles, all spin-dependent contributions are cancelled and the cross section for the combined process of production and decay is given by

$$
\begin{aligned}
\sigma= & \sigma\left(g g \rightarrow \tilde{t}_{1} \overline{\tilde{t}}_{1}\right) \times B R\left(\tilde{t}_{1} \rightarrow t \tilde{\chi}_{2}^{0}\right) \times B R\left(\tilde{\chi}_{2}^{0} \rightarrow \tilde{\chi}_{1}^{0} \ell^{+} \ell^{-}\right) \\
& \times B R(t \rightarrow W b) \\
= & \frac{\left|\Delta\left(\tilde{t}_{1}\right)\right|^{2}\left|\Delta\left(\tilde{\chi}_{2}^{0}\right)\right|^{2}|\Delta(t)|^{2}}{2 E_{b}^{2}} \\
& \times \int P\left(\tilde{t}_{1} \tilde{t}_{1}\right) P\left(\tilde{\chi}_{2}^{0} t\right) D\left(\tilde{\chi}_{2}^{0}\right) D(t)(2 \pi)^{4} \delta^{4} \\
& \times\left(p_{1}+p_{2}-\sum_{n} p_{n}\right) d \operatorname{lips}\left(p_{n}\right) .
\end{aligned}
$$

The explicit expression for the phase space in the laboratory system is given in Appendix C.

\subsection{Structure of the T-odd asymmetries}

Suitable tools to study CP-violating effects are T-odd observables based on triple products of momenta or spin vec- 
Fig. 2.2 Feynman diagrams for the three-body decays $\tilde{\chi}_{i}^{0} \rightarrow \tilde{\chi}_{k}^{0} \ell^{+} \ell^{-}$

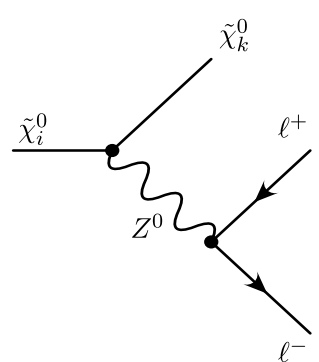

tors of the involved particles. In this paper we study the following T-odd observables:

$$
\begin{aligned}
\mathcal{T}_{t} & =\vec{p}_{t} \cdot\left(\vec{p}_{\ell^{+}} \times \vec{p}_{\ell^{-}}\right), \\
\mathcal{T}_{b} & =\vec{p}_{b} \cdot\left(\vec{p}_{\ell^{+}} \times \vec{p}_{\ell^{-}}\right), \\
\mathcal{T}_{t b} & =\vec{p}_{t} \cdot\left(\vec{p}_{b} \times \vec{p}_{\ell^{ \pm}}\right) .
\end{aligned}
$$

The T-odd asymmetries are defined as

$$
\begin{aligned}
\mathcal{A}_{T_{f}} & =\frac{N_{\mathcal{T}_{f}+}-N_{\mathcal{T}_{f}-}}{N_{\mathcal{T}_{f}+}+N_{\mathcal{T}_{f}-}}=\frac{\int \operatorname{sign}\left\{\mathcal{T}_{f}\right\}|T|^{2} d \text { lips }}{\int|T|^{2} d \text { lips }}, \\
f & =t, b \text { and } t b,
\end{aligned}
$$

where $N_{\mathcal{T}_{f}+}, N_{\mathcal{T}_{f}-}$ are the numbers of events for which $\mathcal{T}_{f}$ is positive and negative respectively, and the second denominator in (2.9), $\int|T|^{2} d$ lips, is proportional to the corresponding cross section, namely $\sigma\left(g g \rightarrow \tilde{t}_{1} \tilde{\bar{t}}_{1} \rightarrow t \tilde{\chi}_{1}^{0} \ell^{+} \ell^{-}\right)$ in (2.6) and $\sigma\left(g g \rightarrow \tilde{t}_{1} \tilde{\bar{t}}_{1} \rightarrow W b \tilde{\chi}_{1}^{0} \ell^{+} \ell^{-}\right)$in (2.7) and (2.8). In the second numerator in (2.9), only the triple-product correlations enter via the spin-dependent terms, as explained in (2.11) and the following sections.

The observable $\mathcal{A}_{T_{b}}$ has the advantage that it is not necessary to reconstruct the momentum of the decaying $t$ quark. However, as explained below, in order to disentangle the effects of both phases of $A_{t}$ and $M_{1}$, it will be necessary to study all possible observables.

The asymmetry $\mathcal{A}_{T_{f}}$, (2.9), is odd under the naïve timereversal operation. It is the difference of the number of events with the final top quark or $b$-jet above and below the plane spanned by $\vec{p}_{\ell^{+}} \times \vec{p}_{\ell^{-}}$in (2.6) and (2.7), and by $\vec{p}_{b} \times \vec{p}_{\ell^{ \pm}}$in (2.8), normalised by the sum of these events.

As can be seen from the numerator of $\mathcal{A}_{T_{f}}$, in order to identify the T-odd contributions, we have to identify those terms in $|T|^{2}$, (2.3), which contain a triple product of the form shown in (2.6)-(2.8). Triple products follow from expressions $i \epsilon_{\mu \nu \rho \sigma} a^{\mu} b^{v} c^{\rho} d^{\sigma}$, where $a, b, c, d$ are 4-momenta and spins of the particles involved, which are non-zero only when the momenta are linearly independent. The expressions $i \epsilon_{\mu \nu \rho \sigma} a^{\mu} b^{v} c^{\rho} d^{\sigma}$ are imaginary and when multiplied by the imaginary parts of the respective couplings they yield terms that contribute to the numerator of $A_{T_{f}}$, (2.9). In our process, T-odd terms with $\epsilon$-tensors are only contained in the spin-dependent contributions to the production,

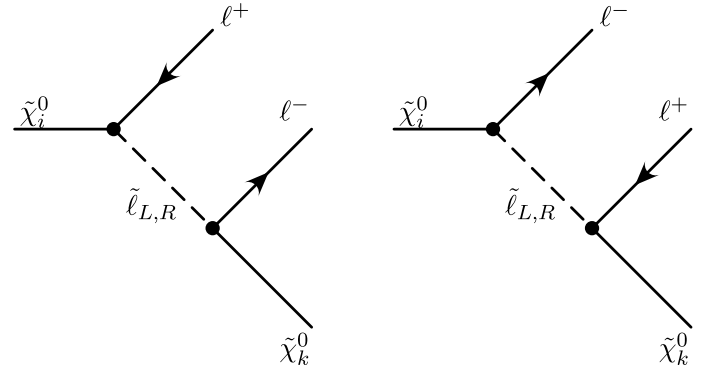

$\Sigma_{P}^{a b}\left(\tilde{\chi}_{j}^{0} t\right)$, and in the spin-dependent terms in neutralino decay, $\Sigma_{D}^{a}\left(\tilde{\chi}_{j}^{0}\right)$. It is therefore convenient to split $\Sigma_{P}^{a b}\left(\tilde{\chi}_{j}^{0} t\right)$ and $\Sigma_{D}^{a}\left(\tilde{\chi}_{j}^{0}\right)$ into T-odd terms $\Sigma_{P}^{a b, \mathrm{O}}\left(\tilde{\chi}_{j}^{0} t\right)$ and $\Sigma_{D}^{a, \mathrm{O}}\left(\tilde{\chi}_{j}^{0}\right)$ containing the respective triple products, and T-even terms $\Sigma_{P}^{a b, \mathrm{E}}\left(\tilde{\chi}_{j}^{0} t\right)$ and $\Sigma_{D}^{a, \mathrm{E}}\left(\tilde{\chi}_{j}^{0}\right)$ without triple products:

$\Sigma_{P}^{a b}\left(\tilde{\chi}_{j}^{0} t\right)=\Sigma_{P}^{a b, \mathrm{O}}\left(\tilde{\chi}_{j}^{0} t\right)+\Sigma_{P}^{a b, \mathrm{E}}\left(\tilde{\chi}_{j}^{0} t\right)$,

$\Sigma_{D}^{a}\left(\tilde{\chi}_{j}^{0}\right)=\Sigma_{D}^{a, \mathrm{O}}\left(\tilde{\chi}_{j}^{0}\right)+\Sigma_{D}^{a, \mathrm{E}}\left(\tilde{\chi}_{j}^{0}\right)$.

The other spin-dependent contributions $\Sigma_{P}^{a}\left(\tilde{\chi}_{j}^{0}\right)$ and $\Sigma_{P}^{b}(t)$, as well as $\Sigma_{D}^{b}(t)$, are T-even.

When multiplying these terms together and composing a T-odd quantity, the only terms of $|T|^{2},(2.3)$, which contribute to the numerator of $\mathcal{A}_{T_{f}}$ are therefore

$$
\begin{aligned}
|T|^{2} \supset & \sum_{a, b=1}^{3}\left[\Sigma_{P}^{a b, \mathrm{O}}\left(\tilde{\chi}_{j}^{0} t\right) \Sigma_{D}^{a, \mathrm{E}}\left(\tilde{\chi}_{j}^{0}\right) \Sigma_{D}^{b}(t)\right. \\
& +\Sigma_{P}^{a, \mathrm{E}}\left(\tilde{\chi}_{j}^{0}\right) \Sigma_{D}^{a, \mathrm{O}}\left(\tilde{\chi}_{j}^{0}\right) \\
& \left.+\Sigma_{P}^{a b, \mathrm{E}}\left(\tilde{\chi}_{j}^{0} t\right) \Sigma_{D}^{a, \mathrm{O}}\left(\tilde{\chi}_{j}^{0}\right) \Sigma_{D}^{b}(t)\right] .
\end{aligned}
$$

The first term in (2.11) is sensitive to the T-odd contributions from the production of the top and the neutralinos $\tilde{\chi}_{j}^{0}$. Comparing (B.15) with (B.27) and (B.40) leads to the following possible combination of contributing momenta

$$
\begin{aligned}
& \Sigma_{P}^{a b, \mathrm{O}}\left(\tilde{\chi}_{j}^{0} t\right) \Sigma_{D}^{a, \mathrm{E}}\left(\tilde{\chi}_{j}^{0}\right) \Sigma_{D}^{b}(t) \\
& \quad \sim \epsilon_{\mu \nu \rho \sigma} s^{a, \mu}\left(\tilde{\chi}_{j}^{0}\right) p_{\tilde{\chi}_{j}^{0}}^{v} s^{b, \rho}(t) p_{t}^{\sigma} \times\left(p_{\left[\ell^{+}, \ell^{-}\right]} s^{a}\right)\left(p_{[b, W]} s^{b}\right) .
\end{aligned}
$$

The second term and third terms in (2.11) are only sensitive to T-odd contributions from the neutralino $\tilde{\chi}_{j}^{0}$ decay. The second term depends only on the polarisation of $\tilde{\chi}_{j}^{0}$, comparing (B.34) with (B.5) therefore leads to the only possible combination of momenta

$$
\Sigma_{P}^{a, \mathrm{E}}\left(\tilde{\chi}_{j}^{0}\right) \Sigma_{D}^{a, \mathrm{O}}\left(\tilde{\chi}_{j}^{0}\right) \sim\left(p_{t} s^{a}\right) \times \epsilon_{\mu \nu \rho \sigma} s^{a \mu} p_{\tilde{\chi}_{j}^{0}}^{v} p_{\ell^{-}}^{\rho} p_{\ell^{+}}^{\sigma}
$$


Since the third term depends on the polarisation of both fermions, $\tilde{\chi}_{j}^{0}$ and $t$, the possible combinations, comparing (B.34) with (B.14) and (B.40), are

$$
\begin{aligned}
& \Sigma_{P}^{a b, \mathrm{E}}\left(\tilde{\chi}_{j}^{0} t\right) \Sigma_{D}^{a, \mathrm{O}}\left(\tilde{\chi}_{j}^{0}\right) \Sigma_{D}^{b}(t) \\
& \quad \sim\left(p_{t} s^{a}\right)\left(p_{\tilde{\chi}_{j}^{0}} s^{b}\right)\left(s^{b} p_{[b, W]}\right) \times \epsilon_{\mu \nu \rho \sigma} s^{a \mu} p_{\tilde{\chi}_{j}^{0}}^{v} p_{\ell^{-}}^{\rho} p_{\ell^{+}}^{\sigma}
\end{aligned}
$$

and $\left(s^{a} s^{b}\right)\left(s^{b} p_{[b, W]}\right) \times \epsilon_{\mu \nu \rho \sigma} s^{a \mu} p_{\tilde{\chi}_{j}^{0}}^{v} p_{\ell^{-}}^{\rho} p_{\ell^{+}}^{\sigma}$.

As can be seen by substituting (B.8)-(B.13) into (B.15) in Appendix B.1, $\Sigma_{P}^{a b, O}\left(\tilde{\chi}_{j}^{0} t\right)$ vanishes for the combinations $(a b)=(11),(22),(33),(13),(31)$, because they contain cross products of three linearly-dependent vectors. Only for the remaining combinations, $(a b)=(12),(21),(23),(32)$, do we get a T-odd contribution to the production density matrix.

Similarly, the expression for the T-even contributions, $\Sigma_{P}^{a b, E}\left(\tilde{\chi}_{j}^{0} t\right),(\mathrm{B} .14)$ in Appendix B.1, has non-zero components for $a=1,3$ but vanishes when $a=2$. These expressions are multiplied by $\Sigma_{D}^{a, \mathrm{O}}\left(\tilde{\chi}_{j}^{0}\right),(\mathrm{B} .34)$, and therefore only $\Sigma_{D}^{1, \mathrm{O}}\left(\tilde{\chi}_{j}^{0}\right)$ and $\Sigma_{D}^{3, \mathrm{O}}\left(\tilde{\chi}_{j}^{0}\right)$ contribute.

In the following section we derive the three triple products, study their different dependence on phases and provide explicitly a strategy for determining $\phi_{A_{t}}$ and $\phi_{M_{1}}$ and disentangling their effects.

\subsection{Strategy for determining $\phi_{A_{t}}$ and $\phi_{M_{1}}$}

\subsubsection{Derivation of the triple products}

In order to describe the spin of a fermion $f$ in general, we introduce three four-vectors, $s_{\mu}^{a}(f), a=1,2,3$, such that the $s^{a}$ and the momentum and mass of the fermion $p / m$ form an orthonormal set of four-vectors [40]; we have

$$
\begin{aligned}
p \cdot s^{a} & =0, \\
s^{a} \cdot s^{b} & =-\delta^{a b}, \\
s_{\mu}^{a} s_{v}^{a} & =-g_{\mu \nu}+\frac{p_{\mu} p_{v}}{m^{2}},
\end{aligned}
$$

where repeated indices are implicitly summed over.

Applying (2.18) on (2.12)-(2.15) lead to kinematic expressions that contain only explicit momenta. Expanding terms with $\epsilon_{\mu \nu \rho \sigma}$ in time- and space- components gives scalar triple products between three momenta.

In our process we can classify the terms of (2.11) as follows.

- The terms of (2.12) lead to a combination between $\mathcal{T}_{t b}$ and $\mathcal{T}_{b}$.

- The terms of (2.13) lead only to $\mathcal{T}_{t}$.
- The terms of (2.14) lead again only to $\mathcal{T}_{t}$ but terms of (2.15) produce $\mathcal{T}_{t}$ as well as $\mathcal{T}_{b}$, due to interference effects between both spin vectors of $p_{t}$ and $p_{\tilde{\chi}_{j}^{0}}$.

\subsubsection{T-odd terms sensitive to $\mathcal{T}_{t}$}

We consider first $\mathcal{T}_{t},(2.6)$. As this includes the reconstructed topquark momentum, there are no spin terms from the decay of the top quark and the contributing terms are the second and third term in (2.11) as explained in the previous paragraph. The $\mathrm{CP}$-sensitive terms of the decay density matrix are given by (B.34)-(B.37) and the contributing kinematical factor is $g_{4}^{a}$, (B.38),

$g_{4}^{a}=i m_{k} \epsilon_{\mu \nu \rho \sigma} s^{a \mu} p_{\tilde{\chi}_{j}^{0}}^{v} p_{\ell^{-}}^{\rho} p_{\ell^{+}}^{\sigma}$.

We note that $g_{4}^{a}$ is purely imaginary. When inserted, for instance, in (B.36), it is multiplied by the factor $i \cdot \operatorname{Im}\left\{f_{\ell j}^{L} f_{\ell k}^{L *} O_{k j}^{\prime \prime} L *\right\}$, which depends on the phases $\phi_{M_{1}}$ (and $\phi_{\mu}$ ) and contributes to $\Sigma_{D}^{a, \mathrm{O}}$. Analogous contributions follow from (B.35) and (B.37). The corresponding Teven terms of the production density matrix also entering in (2.11) are obtained from (B.5).

\subsubsection{T-odd terms sensitive to $\mathcal{T}_{t b}$}

For the triple product $\mathcal{T}_{t b},(2.8)$, only the first term in (2.11) contributes, but the kinematics is complicated by the fact that we need to include the decay of the $t$ in addition to that of the $\tilde{\chi}_{2}^{0}$. This comes from the fact that the kinematical term that generates the triple product is $f_{4}^{a b}$, (B.16):

$f_{4}^{a b}=\epsilon_{\mu \nu \rho \sigma} s^{a, \mu}\left(\tilde{\chi}_{j}^{0}\right) p_{\tilde{\chi}_{j}^{0}}^{v} s^{b, \rho}(t) p_{t}^{\sigma}$.

As both $s^{a, \mu}\left(\tilde{\chi}_{j}^{0}\right)$ and $s^{b, \rho}(t) p_{t}^{\sigma}$ are contained in this term, we need to include their decays in order to produce a nonzero contribution.

This term occurs only once in the $\tilde{t}$ decay amplitude, (B.15), and is multiplied by the complex pre-factor $g^{2} \operatorname{Im}\left(a_{i j} b_{i j}^{*}\right),\left(\right.$ B.17). Both $a_{i j}$ and $b_{i j}$ contain terms from the $\tilde{t}$ and $\tilde{\chi}_{j}^{0}$ mixing matrices, and so are sensitive to both the phases $\phi_{A_{t}}$ and $\phi_{M_{1}}$ (and $\phi_{\mu}$ ).

\subsubsection{T-odd terms sensitive to $\mathcal{T}_{b}$}

The triple product $\mathcal{T}_{b},(2.7)$, is the most complicated, as it contains contributions from both the $\tilde{t}$ and $\tilde{\chi}_{2}^{0}$ decays (the first and third terms in (2.11)). The kinematics is rendered more complex by the need to multiply each T-odd contribution by the terms from the other two decays. Each T-odd component is generated through $g_{4}^{a}$ and $f_{4}^{a b}$, as for the other two triple products. As a consequence of having a dependence on both the $\tilde{t}$ and $\tilde{\chi}_{2}^{0}$ decays, $\mathcal{T}_{b}$ is also sensitive to both phases $\phi_{A_{t}}$ and $\phi_{M_{1}}$ (and $\phi_{\mu}$ ). 


\subsubsection{Disentangling of effects of $\phi_{A_{t}}$ and $\phi_{M_{1}}$}

The T-odd asymmetries, (2.9), are determined by those CPviolating couplings that are multiplied with the respective triple product. Under the assumption that $\phi_{\mu}$ is small, the neutralino sector depends only on $\phi_{M_{1}}$ and the stop sector only on $\phi_{A_{t}}$. Since the involved triple-product momenta show different dependence on the CP-violating phases, as discussed above, it is possible in principle to disentangle the effects of $\phi_{A_{t}}$ and $\phi_{M_{1}}$ in our process and to determine the phases separately.

The decoupling is possible as the triple product $\mathcal{T}_{t}$, can only be produced by the term, $\Sigma_{P}^{a, \mathrm{E}}\left(\tilde{\chi}_{j}^{0}\right) \Sigma_{D}^{a, \mathrm{O}}\left(\tilde{\chi}_{j}^{0}\right)$; cf. Sect. 2.4.1. The T-odd contribution in this term comes from the decay of the $\tilde{\chi}_{j}^{0}$ and consequently is only sensitive to the phase $\phi_{M_{1}}$. Once we have used the triple product $\mathcal{T}_{t}$ to determine the phase $\phi_{M_{1}}$ we can then use the value as an input for the triple products, $\mathcal{T}_{t b}$ and $\mathcal{T}_{b}$, in order to determine the phase $\phi_{A_{t}}$.

\section{Results}

\subsection{Scenarios}

In this section we analyse numerically the various tripleproduct asymmetries introduced in (2.6)-(2.8) at both the parton level and with the inclusion of parton distribution functions (pdfs) to study the discovery potential at the LHC. In particular, we study the dependences of these triple-product asymmetries on the MSSM parameters $M_{1}=$ $\left|M_{1}\right| e^{i \phi_{M_{1}}}$ and $A_{t}=\left|A_{t}\right| e^{i \phi_{A_{t}}}$. We also analyse the effects of these parameters on the masses and branching ratios of the particles involved in our process.

For our numerical analysis we study in detail at both the partonic and pdf level a reference scenario, A, where the $\tilde{\chi}_{1}^{0}$ is a gaugino-higgsino mixture. For comparison, we also study at the partonic level a non-universal Higgs masses (NUHM) scenario, B, and a third scenario, C, in which the $\tilde{\chi}_{2}^{0}$ is higgsino-like. The particle spectra for these scenarios have been computed with the program SPheno [44]. These three scenarios have been chosen to have similar masses, as displayed in Table 3.1, so that the kinematic effects are similar in each case. We perform our studies using our own program based on the analytic formulae we have derived for the various cross sections and spin correlations. The program uses the VEGAS $[45,46]$ routine to perform the multidimensional phase-space integral. We constrain ourselves to cases where $m_{\tilde{\chi}_{2}^{0}}<m_{\tilde{\chi}_{1}^{0}}+m_{Z^{0}}$ and $m_{\tilde{\chi}_{2}^{0}}<m_{\tilde{\ell}_{L, R}}$, so as to forbid the two-body decay of the $\tilde{\chi}_{2}^{0}$. The branching ratios for both processes have been calculated with Herwig ++ [47, 48]. ${ }^{2}$

The feasibility of measuring these asymmetries at the LHC depends heavily on the integrated luminosity at the LHC. For this reason we look closely at the cross section, $\sigma=\sigma\left(g g \rightarrow \tilde{t}_{1} \overline{\tilde{t}}_{1}\right) \times B R\left(\tilde{t}_{1} \rightarrow t \tilde{\chi}_{2}^{0}\right) \times B R\left(\tilde{\chi}_{2}^{0} \rightarrow \tilde{\chi}_{1}^{0} \ell^{+} \ell^{-}\right) \times$ $B R(t \rightarrow W b)$ and determine the nominal luminosity required to observe a statistically significant result.

\subsection{CP asymmetry at the parton level}

\subsubsection{Dependence of $m_{\tilde{\chi}_{1}^{0}}$ and $\mathcal{A}_{T}$ on $\phi_{M_{1}}$ and $\phi_{A_{t}}$}

We start by discussing the dependence on $M_{1}=\left|M_{1}\right| e^{i \phi_{M_{1}}}$ of the parton-level asymmetries for each of the three scenarios. In order to see the maximum dependence upon $\phi_{M_{1}}$, we use the reconstructed $t$ quark momentum and the triple product $\mathcal{T}_{t}=\vec{p}_{t} \cdot\left(\vec{p}_{\ell^{+}} \times \vec{p}_{\ell^{-}}\right)$. It should be noted from the following plots that the asymmetry is obviously a CP-odd quantity that in addition to a measurement of the phase, also gives the sign, as seen in Fig. 3.1(a). In comparison, using CP-even quantities, for example the mass, it is not possible to determine if the phase is positive or negative, as seen in Fig. 3.1(b).

We see in Fig. 3.1(a) that the biggest asymmetry appears in scenario $\mathrm{A}$, which attains $\left|\mathcal{A}_{T_{t}}\right|_{\max } \approx 12 \%$ when $\phi_{M_{1}} \approx 0.3 \pi$. One aspect of the plot that may be surprising is that the asymmetry is not largest at the maximal value of the phase $\left(\phi_{M_{1}}=\frac{\pi}{2}\right)$. This is due to the coupling combinations and interferences and can be seen from the equations in Sect. 2.4.2. In Fig. 3.1(b), the dependence of the masses of the neutralinos is shown. It can be seen clearly that the variations are too small to be used to determine the phase.

In the cases of the two other scenarios shown in Fig. 3.1(a), the dependence of the asymmetry on the phase $\phi_{M_{1}}$ is similar but slightly smaller. In the case of scenario B (NUHM), the peak asymmetry is $\left|\mathcal{A}_{T_{t}}\right|_{\max } \approx 9 \%$ when $\phi_{M_{1}} \approx 0.3 \pi$ and in scenario $\mathrm{C}$ (Higgsino) it is $\left|\mathcal{A}_{T_{t}}\right|_{\max } \approx$ $7 \%$ when $\phi_{M_{1}} \approx 0.25 \pi$. Again, the asymmetry does not peak when the phase is maximal.

To study the dependence upon $\phi_{A_{t}}$ we need to use the triple products sensitive to this phase, $\mathcal{T}_{b}=\vec{p}_{t} \cdot\left(\vec{p}_{\ell^{+}} \times \vec{p}_{\ell^{-}}\right)$ and $\mathcal{T}_{t b}=\vec{p}_{t} \cdot\left(\vec{p}_{b} \times \vec{p}_{\ell^{ \pm}}\right)$. Figure 3.2(a) shows $\mathcal{A}_{\mathcal{T}_{b}}$ and we see that the biggest asymmetry again occurs in Scenario A, but the maximal asymmetry is only about half of $\left|\mathcal{A}_{\mathcal{T}_{t}}\right|_{\max }$ with $\left|\mathcal{A}_{\mathcal{T}_{b}}\right|_{\max } \approx 6 \%$. Scenario $\mathrm{C}$ produces a very similar asymmetry to Scenario A, with $\left|\mathcal{A}_{\mathcal{T}_{b}}\right|_{\max } \approx 5.5 \%$, whereas

\footnotetext{
${ }^{2}$ Beyond the Standard Model physics was produced using the algorithm of [49] and, in the running of $\alpha_{E M}$, the parameterisation of [50] was used.
} 
Table 3.1 Parameters and spectra for the three scenarios A, B, C considered in this paper. We display the input parameters $\left|M_{1}\right|, M_{2}$, $|\mu|, \tan \beta, m_{\tilde{\ell}_{L}}$ and $m_{\tilde{\ell}_{R}}$ and the resulting masses $m_{\tilde{\chi}_{i}^{0}}, i=1, \ldots, 4$, for $\left(\phi_{M_{1}}, \phi_{\mu}\right)=(0.5 \pi, 0)$ and $(0.5 \pi, 0.5 \pi)$. The parameters $M_{2},|\mu|$ and $\tan \beta$ in scenario B are chosen as for the scenario SPS1a in [41]. We used $m_{t}=171.2 \mathrm{GeV}$ [42] and the SM value for the top width $\Gamma_{t} \sim 1.5 \mathrm{GeV}$ [43] for our study. All masses and widths are given in $\mathrm{GeV}$

\begin{tabular}{|c|c|c|c|c|c|c|}
\hline Scenario & \multicolumn{2}{|c|}{ A: Reference } & \multicolumn{2}{|c|}{ B: NUHM - $\gamma$} & \multicolumn{2}{|c|}{ C: Higgsino } \\
\hline$M_{1}$ & \multicolumn{2}{|c|}{109} & \multicolumn{2}{|c|}{97.6} & \multicolumn{2}{|c|}{105} \\
\hline$M_{2}$ & \multicolumn{2}{|c|}{240} & \multicolumn{2}{|c|}{184} & \multicolumn{2}{|c|}{400} \\
\hline$\mu$ & \multicolumn{2}{|c|}{220} & \multicolumn{2}{|c|}{316} & \multicolumn{2}{|c|}{-190} \\
\hline $\tan \beta$ & \multicolumn{2}{|c|}{10} & \multicolumn{2}{|c|}{20} & \multicolumn{2}{|c|}{20} \\
\hline$M_{L}$ & \multicolumn{2}{|c|}{298} & \multicolumn{2}{|c|}{366} & \multicolumn{2}{|c|}{298} \\
\hline$M_{E}$ & \multicolumn{2}{|c|}{224} & \multicolumn{2}{|c|}{341.7} & \multicolumn{2}{|c|}{224} \\
\hline$M_{Q 3}$ & \multicolumn{2}{|c|}{511} & \multicolumn{2}{|c|}{534.5} & \multicolumn{2}{|c|}{511} \\
\hline$M_{U 3}$ & \multicolumn{2}{|c|}{460} & \multicolumn{2}{|c|}{450} & \multicolumn{2}{|c|}{460} \\
\hline$A_{t}$ & \multicolumn{2}{|c|}{-610} & \multicolumn{2}{|c|}{-451.4} & \multicolumn{2}{|c|}{-610} \\
\hline$M_{\tilde{t}_{1}}$ & \multicolumn{2}{|c|}{396.5} & \multicolumn{2}{|c|}{447.8} & \multicolumn{2}{|c|}{402.6} \\
\hline$M_{\tilde{t}_{2}}$ & \multicolumn{2}{|c|}{595} & & & & \\
\hline$M_{\tilde{\chi}_{1}^{ \pm}}$ & & & & & & \\
\hline$M_{\tilde{\chi}_{2}^{ \pm}}$ & & & & & & \\
\hline$m_{\tilde{\ell}_{L}}$ & & & & & & \\
\hline$m_{\tilde{\ell}_{R}}$ & & & & & & \\
\hline$\phi_{M_{1}}$ & 0 & $\pi$ & 0 & $\pi$ & 0 & $\pi$ \\
\hline$m_{\tilde{\chi}_{1}^{0}}$ & 100.8 & 106.1 & 94.8 & 96.3 & 99.2 & 97.6 \\
\hline$m_{\tilde{\chi}_{2}^{0}}$ & 177.0 & 171.3 & 167.1 & 166.6 & 186.2 & 179.8 \\
\hline$m_{\tilde{\chi}_{3}^{0}}$ & 227.9 & 231.8 & 323.8 & 325.5 & 199.4 & 206.2 \\
\hline$m_{\tilde{\chi}_{4}^{0}}$ & 299.1 & 297.6 & 343.4 & 341.8 & 419 & 418.9 \\
\hline$\Gamma_{\tilde{t}_{1}}$ & 3.88 & 3.88 & 3.48 & 3.48 & 5.29 & 5.29 \\
\hline$\Gamma_{\tilde{\chi}_{2}^{0}}$ & $1.4 \times 10^{-4}$ & $1.4 \times 10^{-4}$ & $2.3 \times 10^{-5}$ & $2.3 \times 10^{-5}$ & $3.0 \times 10^{-3}$ & $3.0 \times 10^{-3}$ \\
\hline
\end{tabular}

Fig. 3.1 The asymmetry at threshold for the production process $g g \longrightarrow \tilde{t} \overline{\tilde{t}}$ for scenarios $\mathrm{A}, \mathrm{B}$ and $\mathrm{C}$ for (a) $\mathcal{A}_{\mathcal{T}_{t}}$ as a function of $\phi_{M_{1}}$, and (b) the masses of the neutralinos as functions of $\phi_{M_{1}}$

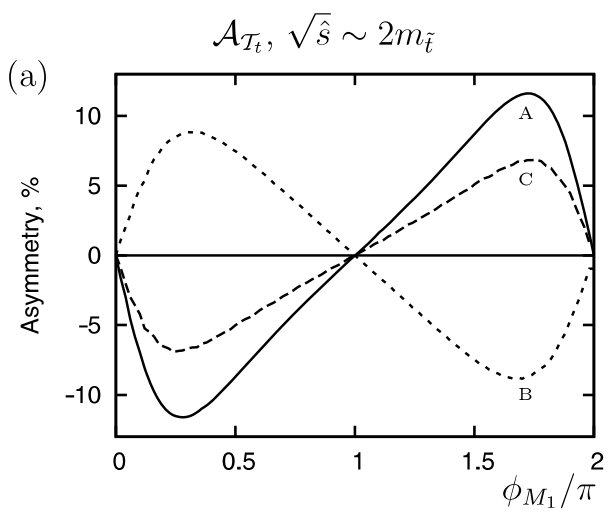

the asymmetry in Scenario B is much smaller: $\left|\mathcal{A}_{\mathcal{T}_{b}}\right|_{\max } \approx$ $2.5 \%$. Figure 3.2(b) shows that the general shape of the asymmetries for $\mathcal{A}_{\mathcal{T}_{t b}}$ is similar to that of $\mathcal{A}_{\mathcal{T}_{b}}$ apart from a difference in sign and that all the asymmetries are actually slightly larger. In fact, for Scenario $\mathrm{C}$, the largest asymmetry is generated using $\mathcal{T}_{t b}$ with $\mathcal{A}_{\mathcal{T}_{t b}} \approx 8 \%$ when $\phi_{A_{t}} \approx 0.3 \pi$.

In the subsequent analysis, we concentrate on the favourable Scenario A, with just a few remarks on the others.

\subsubsection{Contour Plots of $\mathcal{A}_{T_{t}}$ and $\mathcal{A}_{T_{t b}}$ for Variable $M_{1}$ and $A_{t}$}

If we now lift the restriction of the GUT relation for $\left|M_{1}\right|$, we can see how the asymmetry varies with $\left|M_{1}\right|$ while leaving all the other parameters the same, for scenario A. Figure 3.3(a) shows that the asymmetry peaks at $\left|M_{1}\right| \approx$ $130 \mathrm{GeV}$ and $\phi_{M_{1}} \approx 0.25 \pi$ when $\left|\mathcal{A}_{T_{t}}\right| \approx 15 \%$. Importantly though, the asymmetry can remain above $10 \%$ be- 
tween $\left|M_{1}\right|=110 \mathrm{GeV}$ and $\left|M_{1}\right|=190 \mathrm{GeV}$, which is most of the range allowed in this scenario.

By including the decay of the $t$ quark that was produced in the $\tilde{t}$ decay, we can also study the effect of $\phi_{A_{t}}$ on our asymmetries. As the spin-correlation information is now carried by the $t$ quark, we have to change the triple product used to measure the asymmetry, (2.9). It is found that the largest asymmetry can be measured using the triple product, $\mathcal{T}_{t b}=\mathbf{p}_{\ell^{+}} \cdot\left(\mathbf{p}_{t} \times \mathbf{p}_{b}\right)$ where $\left|\mathcal{A}_{T_{t b}}\right|_{\max } \approx 8.5 \%$ when $\phi_{A_{t}} \approx 0.5 \pi$ in Scenario A, as seen in Fig. 3.3(a). It may be noted that this asymmetry is slightly smaller than those of [21] that can be reconstructed experimentally. In that paper scenarios were chosen where the $\tilde{\chi}_{2}^{0}$ decays via a two-body process, whereas here we concentrate on scenarios where the $\tilde{\chi}_{2}^{0}$ decays via a three-body process, so to maximise the sensitivity to $\phi_{M_{1}}$. This phase dependence can also be seen with the triple product $\mathcal{T}_{b}=\mathbf{p}_{b} \cdot\left(\mathbf{p}_{\ell^{+}} \times \mathbf{p}_{\ell^{+}}\right)$although the asymmetry is found to be smaller here with $\left|\mathcal{A}_{T_{b}}\right|_{\max } \approx 6 \%$; see Fig. 3.4(a).

We have also considered the dependence of the asymmetry on a common trilinear coupling, $A=A_{t}=A_{b}=A_{\tau}$, in scenario A, as shown in Fig. 3.4(b). It can be seen that the asymmetry is stable for the bulk of the region scanned, and only decreases near the edge of the acceptable region for our scenario. The peak is now $\left|\mathcal{A}_{T_{t b}}\right|_{\max } \approx 9 \%$, when $A_{t} \approx-500 \mathrm{GeV}$, and the region where $\left|\mathcal{A}_{T_{t b}}\right|>8 \%$ extends from $A_{t} \approx-650 \mathrm{GeV}$ to $A_{t} \approx-250 \mathrm{GeV}$.

We now consider the effect on the asymmetry of varying simultaneously both the phases $\phi_{M_{1}}$ and $\phi_{A_{t}}$. The triple products $\mathcal{T}_{b}=\vec{p}_{b} \cdot\left(\vec{p}_{\ell^{+}} \times \vec{p}_{\ell^{-}}\right)$and $\mathcal{T}_{t b}=\vec{p}_{t} \cdot\left(\vec{p}_{b} \times \vec{p}_{\ell^{ \pm}}\right)$ can have contributions from both phases, so we concentrate on these. For $\mathcal{T}_{b}$, Fig. 3.5(a) shows that the area of parameter
Fig. 3.2 (a) The asymmetry $\mathcal{A}_{\mathcal{T}_{b}}$ at threshold for the production process $g g \longrightarrow \tilde{t} \overline{\tilde{t}}$ for scenarios A, B and C, and (b) the asymmetry $\mathcal{A}_{\mathcal{T}_{b}}$ at threshold, both as functions of $\phi_{A_{t}}$
Fig. 3.3 Contours in scenario A (in \%) of the parton-level asymmetries (a) $\mathcal{A}_{T_{t}}$ for the triple product

$\mathcal{T}_{t}=\mathbf{p}_{t} \cdot\left(\mathbf{p}_{\ell^{+}} \times \mathbf{p}_{\ell^{-}}\right)$, as functions of the variables $M_{1}$ and $\phi_{M_{1}}$, and (b) $\mathcal{A}_{T_{t b}}$ for the triple product

$\mathcal{T}_{t b}=\mathbf{p}_{\ell^{+}} \cdot\left(\mathbf{p}_{t} \times \mathbf{p}_{b}\right)$, as functions of the variables $M_{1}$ and $\phi_{A_{t}}$

Fig. 3.4 Contours in scenario A (in \%) of the asymmetries (a) $\mathcal{A}_{b}$ for the triple product $\mathcal{T}=\mathbf{p}_{b} \cdot\left(\mathbf{p}_{\ell^{-}} \times \mathbf{p}_{\ell^{+}}\right)$, as functions of the variables $M_{1}$ and $\phi_{A_{t}}$ and (b) $\mathcal{A}_{t b}$ for the triple product

$\mathcal{T}=\mathbf{p}_{\ell^{+}} \cdot\left(\mathbf{p}_{t} \times \mathbf{p}_{b}\right)$, as functions of the common variables $A=A_{t}=A_{b}=A_{\tau}$ and $\phi_{A_{t}}$
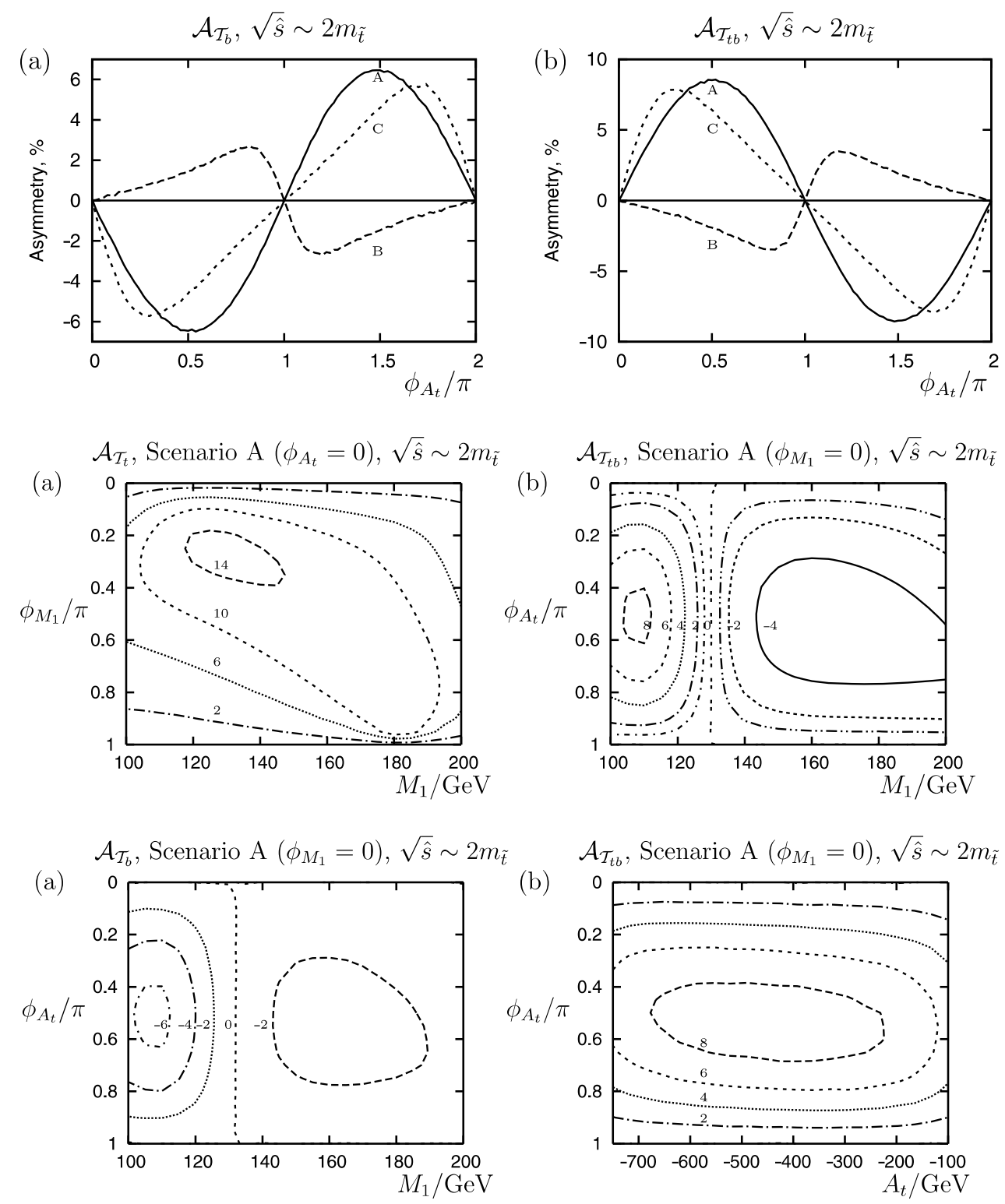
space where $\phi_{M_{1}}$ and $\phi_{A_{t}}$ constructively interfere is actually quite small and peaked around $\phi_{M_{1}} \approx 0.2 \pi$ and $\phi_{A_{t}} \approx 0.5 \pi$. Apart from this area, varying both phases generally results in a reduction in the asymmetry observed, caused by the neutralino and squark mixing entering the couplings, Sect. 2.4.3. Importantly when $\phi_{M_{1}} \approx \pi$ or $\phi_{A_{t}} \approx \pi$ the asymmetry vanishes, as it should. Figure 3.5(b) demonstrates that, for this scenario, $\phi_{M_{1}}$ generates virtually no asymmetry for $\mathcal{T}_{b}$. However, $\phi_{M_{1}}$ can still significantly reduce the asymmetry that $\phi_{A_{t}}$ can produce and, again, when $\phi_{M_{1}} \approx \pi$ we see that $\left|\mathcal{A}_{T_{t b}}\right| \approx 0$ as expected.

If we now modify scenario A slightly by setting $\left|M_{1}\right|=$ $160 \mathrm{GeV}$, this results in a more interesting scenario as the phases $\phi_{M_{1}}$ and $\phi_{A_{t}}$ can interfere constructively to produce an asymmetry larger than that seen before. When
$\phi_{M_{1}} \approx 0.4 \pi$ and $\phi_{A_{t}} \approx 1.8 \pi$, we observe a peak asymmetry, $\left|\mathcal{A}_{T_{b}}\right| \approx 7 \%$ for the triple product $\mathcal{T}_{b}$, as seen in Fig. 3.6.

\subsection{Dependences of branching ratios on $\phi_{M_{1}}$ and $\phi_{A_{t}}$}

In order to determine whether an asymmetry could be observed at the LHC, we need to calculate the cross section for the total process. Important factors in the total cross section are the branching ratios $B R\left(\tilde{t}_{1} \rightarrow \tilde{\chi}_{2}^{0} t\right)$ (for CP-violating case see [51]) and $B R\left(\tilde{\chi}_{2}^{0} \rightarrow \tilde{\chi}_{1}^{0} \ell^{+} \ell^{-}\right)$[38]. Both of these change considerably with $\phi_{M_{1}}$ and $\phi_{A_{t}}$, altering the statistical significance of any measurement of $\left|\mathcal{A}_{T_{f}}\right|$. Analysing first the variation with $M_{1}$, seen in Figs. 3.7 and 3.8(a), we see that the branching ratio $B R\left(\tilde{t}_{1} \rightarrow \tilde{\chi}_{2}^{0} t\right)$ is indeed sensitive to variation of the phase but can vary more strongly with $\left|M_{1}\right|$. For example, if $\phi_{M_{1}}=\pi$ when $\left|M_{1}\right| \approx 150 \mathrm{GeV}$ then
Fig. 3.5 Contours (in \%) of the asymmetry at the parton level in scenario A with $M_{1}=109 \mathrm{GeV}$ for the triple products

(a) $\mathcal{T}_{b}=\mathbf{p}_{b} \cdot\left(\mathbf{p}_{\ell^{+}} \times \mathbf{p}_{\ell^{-}}\right)$and

(b) $\mathcal{T}_{t b}=\mathbf{p}_{\ell^{+}} \cdot\left(\mathbf{p}_{b} \times \mathbf{p}_{t}\right)$ for

varying phases $\phi_{M_{1}}$ and $\phi_{A_{t}}$

Fig. 3.6 Contours (in \%) of the asymmetries in scenario $\mathrm{A}$ at the parton level with

$M_{1}=160 \mathrm{GeV}$ for the triple products

(a) $\mathcal{T}_{b}=\mathbf{p}_{b} \cdot\left(\mathbf{p}_{\ell^{+}} \times \mathbf{p}_{\ell^{-}}\right)$and

(b) $\mathcal{T}_{t b}=\mathbf{p}_{\ell^{+}} \cdot\left(\mathbf{p}_{b} \times \mathbf{p}_{t}\right)$, as

functions of the varying phases

$\phi_{M_{1}}$ and $\phi_{A_{t}}$

Fig. 3.7 Contours (in \%) of branching ratios in scenario $\mathrm{A}$ as functions of $M_{1}$ and $\phi_{M_{1}}$ :
(a) $B R\left(\tilde{t}_{1} \rightarrow \tilde{\chi}_{2}^{0} t\right)$ and
(b) $B R\left(\tilde{\chi}_{2}^{0} \rightarrow \tilde{\chi}_{1}^{0} \ell^{+} \ell^{-}\right), \ell=e$
or $\mu$
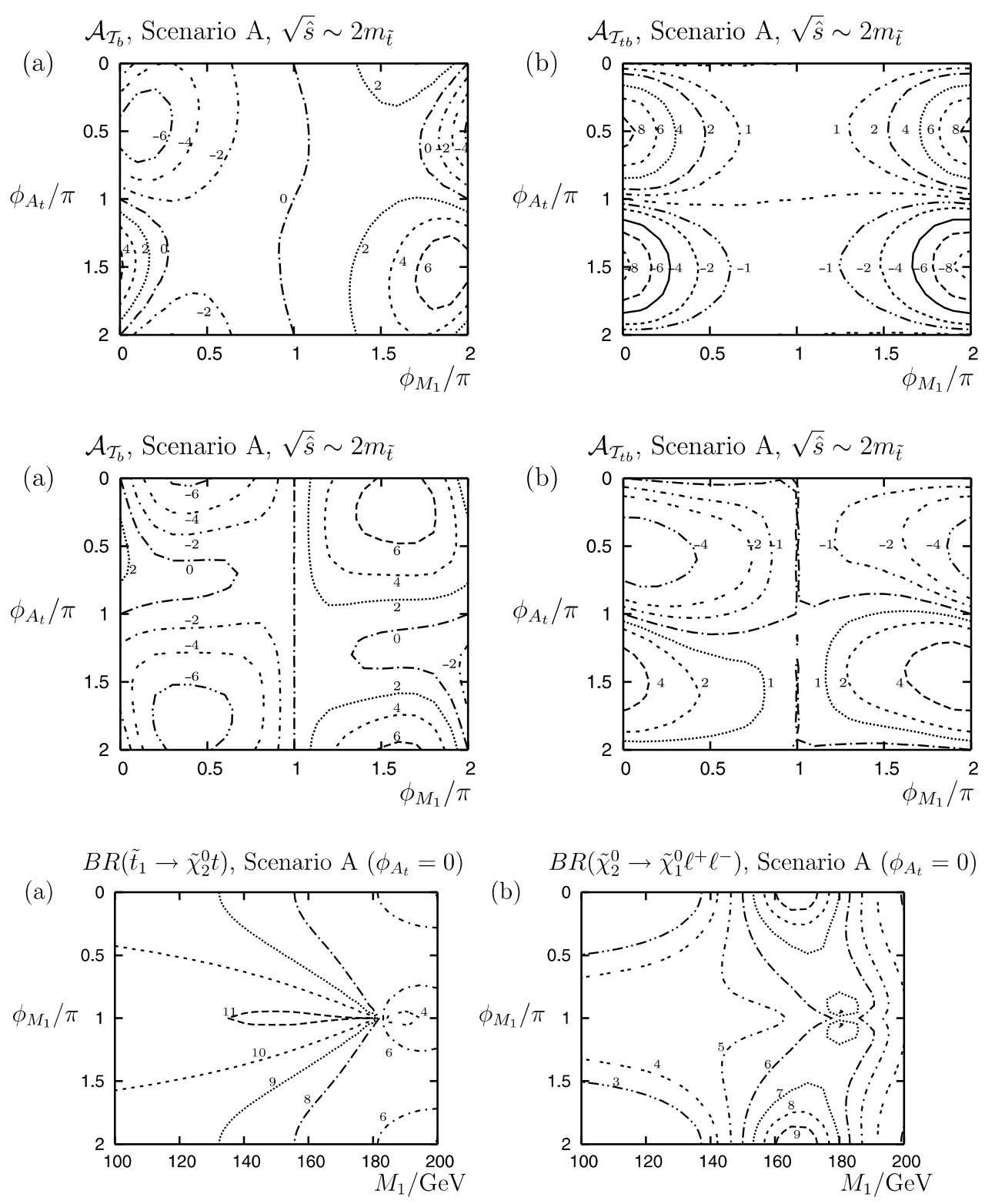
$B R \approx 11 \%$, but if we keep the phase the same and change to $\left|M_{1}\right| \approx 180 \mathrm{GeV}$ then $B R \approx 4 \%$ (i.e., it drops by almost a factor of four), as seen in Fig. 3.7(a). The general reduction of $B R\left(\tilde{t}_{1} \rightarrow \tilde{\chi}_{2}^{0} t\right)$ as $M_{1}$ increases is to be expected as the character of $\tilde{\chi}_{2}^{0}$ will be less gaugino-like. Similar large differences are found in $B R\left(\tilde{\chi}_{2}^{0} \rightarrow \tilde{\chi}_{1}^{0} \ell^{+} \ell^{-}\right)$which varies between $3 \%$ for $M_{1}<135 \mathrm{GeV}$ and $9 \%$ for $M_{1} \approx 165 \mathrm{GeV}$ Fig. 3.7(b).

The phase $\phi_{A_{t}}$ does not enter $B R\left(\tilde{\chi}_{2}^{0} \rightarrow \tilde{\chi}_{1}^{0} \ell^{+} \ell^{-}\right)$, but it can have a large effect on $B R\left(\tilde{t}_{1} \rightarrow \tilde{\chi}_{2}^{0} t\right)$. In scenario A, we see in Fig. 3.8 that $B R \approx 8 \%$ at $\phi_{A_{t}}=0$ but increases to $B R \approx 24 \%$ at $\phi_{A_{t}}=\pi$ (i.e. a factor of 3 increase). The branching ratio $B R\left(\tilde{t}_{1} \rightarrow \tilde{\chi}_{2}^{0} t\right)$ also has a dependence on $\left|A_{t}\right|$ an this is shown in Fig. 3.8(b). We see that if $\phi_{A_{t}}=0$ then the branching vary between, $B R \approx 4 \%$ when $\left|A_{t}\right| \approx-750 \mathrm{GeV}$ and $B R \approx 12 \%$ when $\left|A_{t}\right| \approx-100 \mathrm{GeV}$.

In the range of $M_{1}=\left|M_{1}\right| e^{i \phi_{M_{1}}}$ and $A_{t}=\left|A_{t}\right| e^{i \phi_{A_{t}}}$ studied, we find that $B R\left(\tilde{t}_{1} \rightarrow \tilde{\chi}_{2}^{0} t\right)$ varies between $4 \%$ and $24 \%$ and $B R\left(\tilde{\chi}_{2}^{0} \rightarrow \tilde{\chi}_{1}^{0} \ell^{+} \ell^{-}\right)$between $2.5 \%$ and $9 \%$ for scenario A. Similar plots can also be produced for scenarios B and $\mathrm{C}$ but are not presented here. It is found that $B R\left(\tilde{t}_{1} \rightarrow \tilde{\chi}_{2}^{0} t\right)$ varies between $4 \%$ and $14 \%$ for scenario $B$ and between $8 \%$ and $35 \%$ for scenario $\mathrm{C}$. For $B R\left(\tilde{\chi}_{2}^{0} \rightarrow \tilde{\chi}_{1}^{0} \ell^{+} \ell^{-}\right)$the variation is between $3 \%$ and $12 \%$ for scenario $\mathrm{B}$ and between $2 \%$ and $5 \%$ for scenario $\mathrm{C}$.
3.4 Influence of parton distribution functions (pdfs) on $\mathrm{CP}$ asymmetries

So far we have studied the triple-product asymmetries only when the production process is close to threshold, and the $\tilde{t}_{1}$ pair is produced almost at rest in its centre-of-mass frame; triple-product effects due to spin effects are usually greatest close to threshold. However, production at the LHC is not in general close to threshold, and we must include pdfs in our analysis to see how an initial boost to the $\tilde{t}_{1}$ affects the asymmetry. We focus on scenario A: similar results are obtained in Scenarios B and C.

Figure 3.9(a) shows the asymmetry $\left|\mathcal{A}_{T_{t}}\right|$ as a function of the $\tilde{t}_{1}$ momentum, and shows clearly that the asymmetry is peaked at the threshold for $\tilde{t}_{1}$ production, where the stops are produced almost at rest, and that it falls sharply as the energy increases. Figure 3.9(b) shows the total cross section in $14 \mathrm{TeV}$ collisions at the LHC for $g g \rightarrow \tilde{t}_{1} \overline{\tilde{t}}_{1}$ as a function of the parton-parton centre-of-mass energy, and it demonstrates that the peak production occurs close to threshold with a long tail of production at high energy. In addition, even when production occurs at a low parton-parton centreof-mass energy, in the majority of cases one gluon may be carrying significantly more momentum than the other in the collision. Consequently the produced $\tilde{t}_{1}$ can have a large longitudinal component to its momenta. Both these factors mean that the asymmetry observed at the LHC will be substantially smaller than if the all $\tilde{t}_{1}$ were produced at thresh-
Fig. 3.8 Contours (in \%) of the branching ratio $B R\left(\tilde{t}_{1} \rightarrow \tilde{\chi}_{2}^{0} t\right)$, in scenario $\mathrm{A}$ as functions of varying (a) $M_{1}$ and $\phi_{A_{t}}$ and (b) the common trilinear coupling $A_{t}=A_{b}=A_{\tau}$ and the phase of the top-quark trilinear coupling $\phi_{A_{t}}$
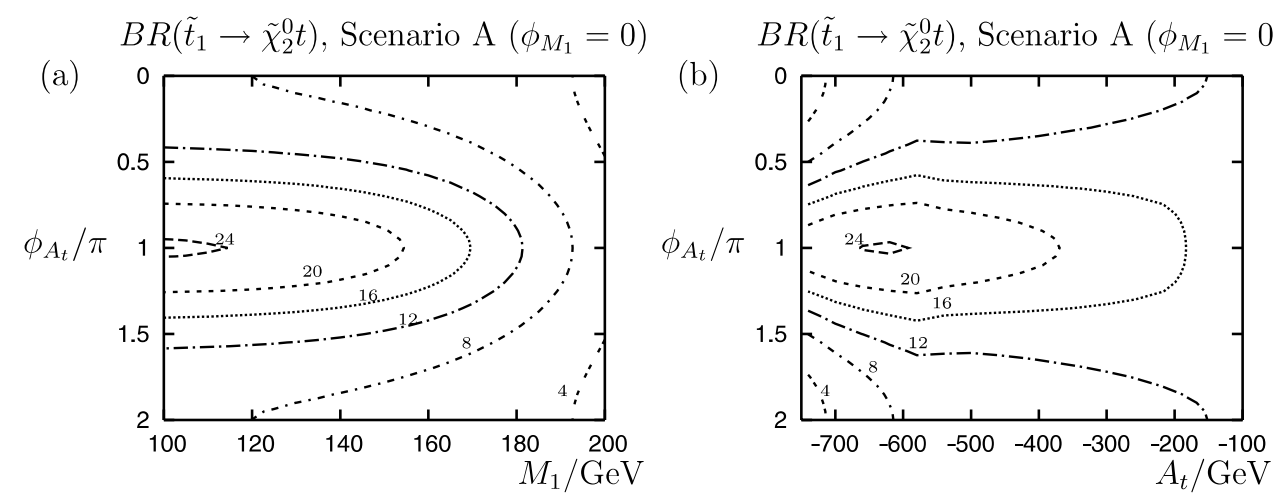

Fig. 3.9 (a) Asymmetry $\mathcal{A}_{T_{t}}$ for scenario $\mathrm{A}$ as a function of the $\tilde{t}$ momentum. (b) Total cross section for scenario A for $g g \longrightarrow \tilde{\tilde{t}} \overline{\tilde{t}}$ as a function of the parton-parton centre-of-mass energy

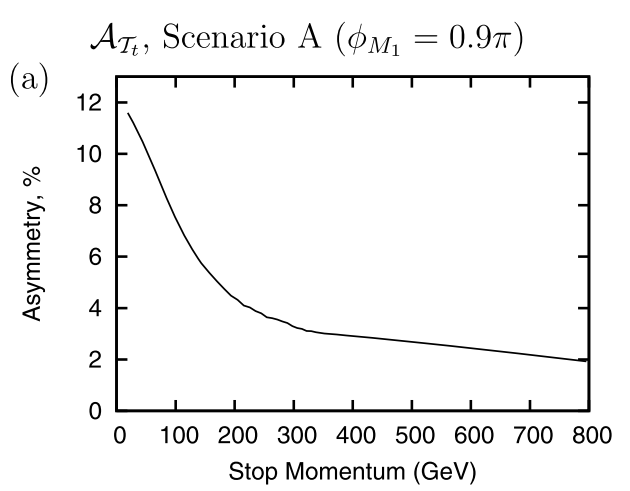


Fig. 3.10 Integrated asymmetries with parton density functions included in the production process. The dotted and dashed lines indicate the asymmetry required in order to observe a $1 \sigma$ deviation from zero with the indicated luminosities; see the text: (a) $\mathcal{T}_{t}=\mathbf{p}_{t} \cdot\left(\mathbf{p}_{\ell^{+}} \times \mathbf{p}_{\ell^{-}}\right)$in scenario $\mathrm{A}$ as a function of $\phi_{M_{1}}$ with $M_{1}=130 \mathrm{GeV}$, and

(b) $\mathcal{T}_{t b}=\mathbf{p}_{\ell^{+}} \cdot\left(\mathbf{p}_{t} \times \mathbf{p}_{b}\right)$ in scenario $\mathrm{A}$ as a function of $\phi_{A_{t}}$ with $M_{1}=109 \mathrm{GeV}$
$\mathcal{A}_{\mathcal{T}_{t}}$, Scenario A $\left(M_{1}=130 \mathrm{GeV}\right.$, $\left.\phi_{A_{t}}=0\right), \sqrt{\hat{s}}=14 \mathrm{TeV}$

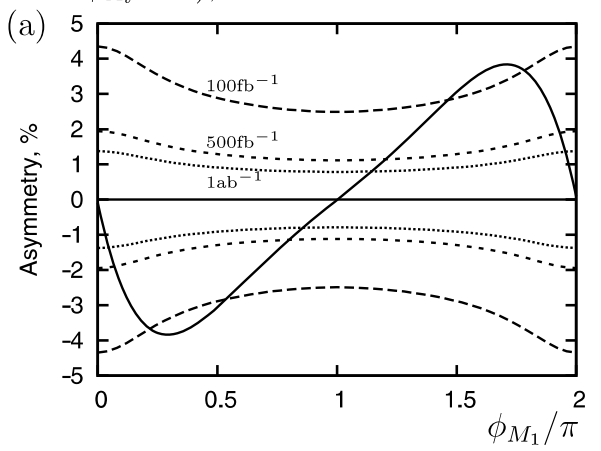

$\mathcal{A}_{\mathcal{T}_{\text {tb }}}$, Scenario A $\left(\phi_{M_{1}}=0\right), \sqrt{\hat{s}}=14 \mathrm{TeV}$

(b)

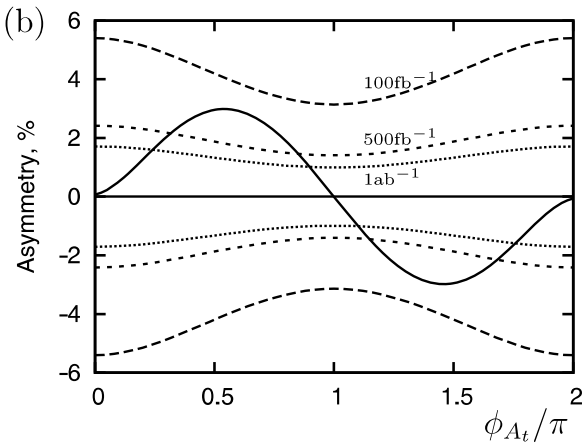

old. ${ }^{3}$ In should be noted that similar results were found for all asymmetries and scenarios, and this 'dilution' factor is always present.

We use the MRST 2004LO pdf set [52] in our analysis of the asymmetry, and plot the integrated asymmetry $\left|\mathcal{A}_{T_{t}}\right|$ as a function of $\phi_{M_{1}}$ and $\phi_{A_{t}}$ at the LHC in Fig. 3.10(a), as the solid line. We see that the inclusion of the pdfs reduces the asymmetry by about a factor of four in this case. This reduction is unsurprising, given the reduction in asymmetry when one moves away from threshold shown in Fig. 3.9(a), though the dilution factor does depend on the scenario. ${ }^{4}$

Using the production cross sections and branching ratios we can then estimate the integrated luminosity required to observe an asymmetry at the LHC. We assume that $N_{\mathcal{T} \pm}$, the numbers of events where $\mathcal{T}$ is positive and negative as in (2.9), are binomially distributed, giving the following statistical error [53]:

$\Delta\left(\mathcal{A}_{T}\right)^{\text {stat }}=2 \sqrt{\epsilon(1-\epsilon) / N}$,

where $\epsilon=N_{\mathcal{T}+} /\left(N_{\mathcal{T}+}+N_{\mathcal{T}-}\right)=\frac{1}{2}\left(1+\mathcal{A}_{T}\right)$, and $N$ is the number of selected events. This can be rearranged to give the required number of events for a desired significance.

Figures 3.10(a), (b) and 3.11(a) show the expected levels of the integrated asymmetries in scenario A with pdf effects included (solid line) together with dotted and dashed lines showing the level of asymmetry one would need with the corresponding integrated luminosity in order to obtain a statistical error $\mathcal{A}_{T}>\Delta\left(A_{T}\right)$. In other words, the asymmetry could only be seen at the level of $1 \sigma$ where the solid line is above the relevant dotted or dashed line. For example, in scenario A after $100 \mathrm{fb}^{-1}$, the asymmetry could only be seen

\footnotetext{
${ }^{3}$ Both these effects could be overcome if one could measure the stopstop invariant mass and tag the stop momenta, but this is unlikely to be possible with great accuracy.

${ }^{4}$ These results have been checked independently using Herwig $++[47,48]$ with three-body spin correlations included, a feature that is currently not available in an official release of the code, but will be included in a future version.
}

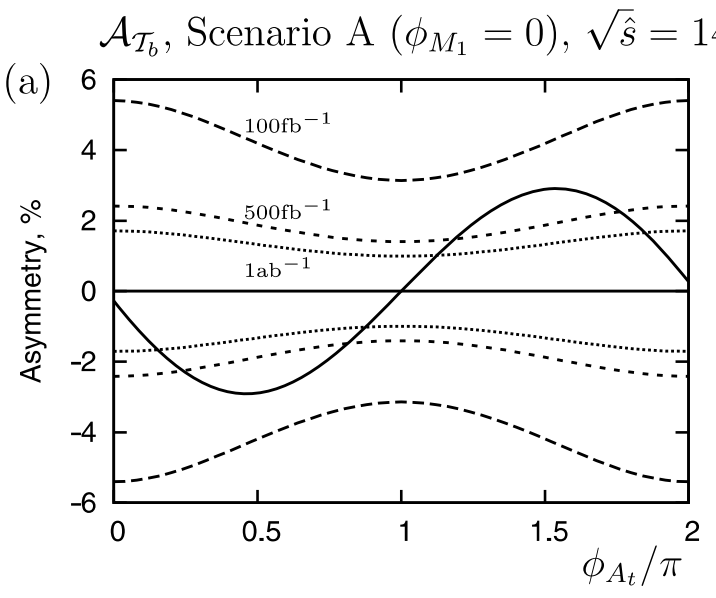

Fig. 3.11 Integrated asymmetries with parton density functions included in the production process. The dotted and dashed lines indicate the asymmetry required in order to observe a $1 \sigma$ deviation from zero with the indicated luminosities; see the text: (a) $\mathcal{T}_{b}=\mathbf{p}_{b} \cdot\left(\mathbf{p}_{\ell^{+}} \times \mathbf{p}_{\ell^{-}}\right)$ in scenario A as a function of $\phi_{A_{t}}$ with $M_{1}=109 \mathrm{GeV}$

for a small area of parameter space around $\phi_{M_{1}}=0.35 \pi$ and $1.7 \pi$. Figures 3.10(a) and (b) show that even if $\phi_{M_{1}}$ or $\phi_{A_{t}}$ has a value that produces a maximal asymmetry, we require a substantial integrated luminosity if we are to find a statistically significant result. In addition, it must be noted that we have not included any detector effects into our analysis, and one could expect that the required integrated luminosity would rise substantially after the inclusion of backgrounds, trigger efficiencies, etc. A measurement of an asymmetry with an accuracy of a few $\%$ might be possible with $100 \mathrm{fb}^{-1}$ of integrated luminosity, but it would probably be insufficient to constrain significantly the model parameter space. However, an interesting measurement could be made with an integrated luminosity above $300 \mathrm{fb}^{-1}$, which is targeted by the proposed LHC luminosity upgrade.

\subsection{Determination of the $\mathrm{CP}$-violating phases}

As we have shown, it will be challenging to determine the phases $\phi_{M_{1}}$ and $\phi_{A_{t}}$ in our process using the triple-product 
asymmetries alone. However, it would be very worthwhile, as a non-zero measurement of a T-odd asymmetry would provide unique evidence of $\mathrm{CP}$ violation. In the rest of this section, we examine briefly the potential for a measurement using other variables, again concentrating on scenario A.

\subsubsection{Observables: masses, cross sections and CP asymmetries}

Figure 3.2(b) showed how the masses of the $\tilde{\chi}_{i}^{0}$ s vary with $\phi_{M_{1}}$ and Fig. 3.12(a) shows how the masses of the $\tilde{t}_{i}$ vary with $\phi_{A_{t}}$ in scenario A. Unfortunately, the variations in both of these observables are only about $1-2 \mathrm{GeV}$, which are significantly smaller than the experimental errors expected for these measurements. A far more accurate measurement at the LHC will be the mass difference between $\tilde{\chi}_{2}^{0}$ and $\tilde{\chi}_{1}^{0}$, as this can be determined in our scenario with a clear dilepton end-point. The accuracy of this measurement is expected to be $<1 \%$ and, if we assume that $M_{2}$ can be determined to 5\% [54], we find the regions plotted in Fig. 3.12(b). At the smaller values allowed for $M_{1}$ in scenario $\mathrm{A}$, we see that this observable does not depend sensitively enough on $\phi_{M_{1}}$ for a measurement to become possible. However, as $M_{1}$ increases we see that the sensitivity to $\phi_{M_{1}}$ becomes much clearer. Importantly, in scenario $\mathrm{A}$, it is only possible to have a mass difference, $\tilde{\chi}_{2}^{0}-\tilde{\chi}_{1}^{0} \lesssim 40 \mathrm{GeV}$ if $\phi_{M_{1}}$ is present.

\subsubsection{Inclusion of branching ratios}

Other observables sensitive to the phases $\phi_{M_{1}}$ and $\phi_{A_{t}}$ are the branching ratios $B R\left(\tilde{\chi}_{2}^{0} \rightarrow \tilde{\chi}_{1}^{0} \ell^{+} \ell^{-}\right)$and $B R\left(\tilde{t}_{1} \rightarrow \tilde{\chi}_{2}^{0} t\right)$, as discussed in Sect. 3.3. As is the case for the masses, though, our current expectation of the accuracy of this measurement at the LHC looks insufficient to constrain the phases. Figure 3.13(a) shows in the context of scenario A that, if a measurement $B R\left(\tilde{\chi}_{2}^{0} \rightarrow \tilde{\chi}_{1}^{0} \ell^{+} \ell^{-}\right)=0.4$ is made and we assume that the accuracy at the LHC is $50 \%\left(\Delta_{1}\right)$, then the constraints on $M_{1}$ and $\phi_{M_{1}}$ are rather weak. However, if the accuracy could be improved to $10 \%\left(\Delta_{2}\right)$, a determination of $M_{1}$ and $\phi_{M_{1}}$ looks possible if this analysis is combined with information from the $\tilde{\chi}_{2}^{0}, \tilde{\chi}_{1}^{0}$ mass difference and that of the triple-product correlations. For the branching ratio, $B R\left(\tilde{t}_{1} \rightarrow \tilde{\chi}_{2}^{0} t\right)$, the conclusion is similar, as seen in Fig. 3.13(b). With a measurement at $50 \%\left(\Delta_{1}\right)$, we again see that a determination of the $\mathrm{CP}$-violating parameter is not possible but, if a measurement can be made with an accuracy of $10 \%\left(\Delta_{2}\right)$, then a determination of $\phi_{A_{t}}$ would be more plausible.

Thus, we may be able to pin the model parameters down with greater accuracy by combining information on the $\mathrm{CP}$ violating asymmetries with this and other information.
Fig. 3.12 (a) The mass of the stop squarks $\tilde{t}_{j}, j=1,2$ as functions of $\phi_{A_{t}} / \pi$, and (b) contour plot showing the areas of the $\left(M_{1}, \phi_{M_{1}}\right)$ parameter plane consistent with a mass difference between $\tilde{\chi}_{2}^{0}$ and $\tilde{\chi}_{1}^{0}$ of 20,40 and $60 \mathrm{GeV}$ respectively. The bands assume a $1 \%$ error in experimental measurement of the mass difference and a $5 \%$ error in $M_{2}$

Fig. 3.13 Parameter space allowed when the experimental accuracy of the branching ratio measurement is $50 \%\left(\Delta_{1}\right)$ or $10 \%\left(\Delta_{2}\right)$ for

(a) $B R\left(\tilde{\chi}_{2}^{0} \rightarrow \tilde{\chi}_{1}^{0} \ell^{+} \ell^{-}\right)=0.04$ and (b) $B R\left(\tilde{t}_{1} \rightarrow \tilde{\chi}_{2}^{0} t\right)=0.1$
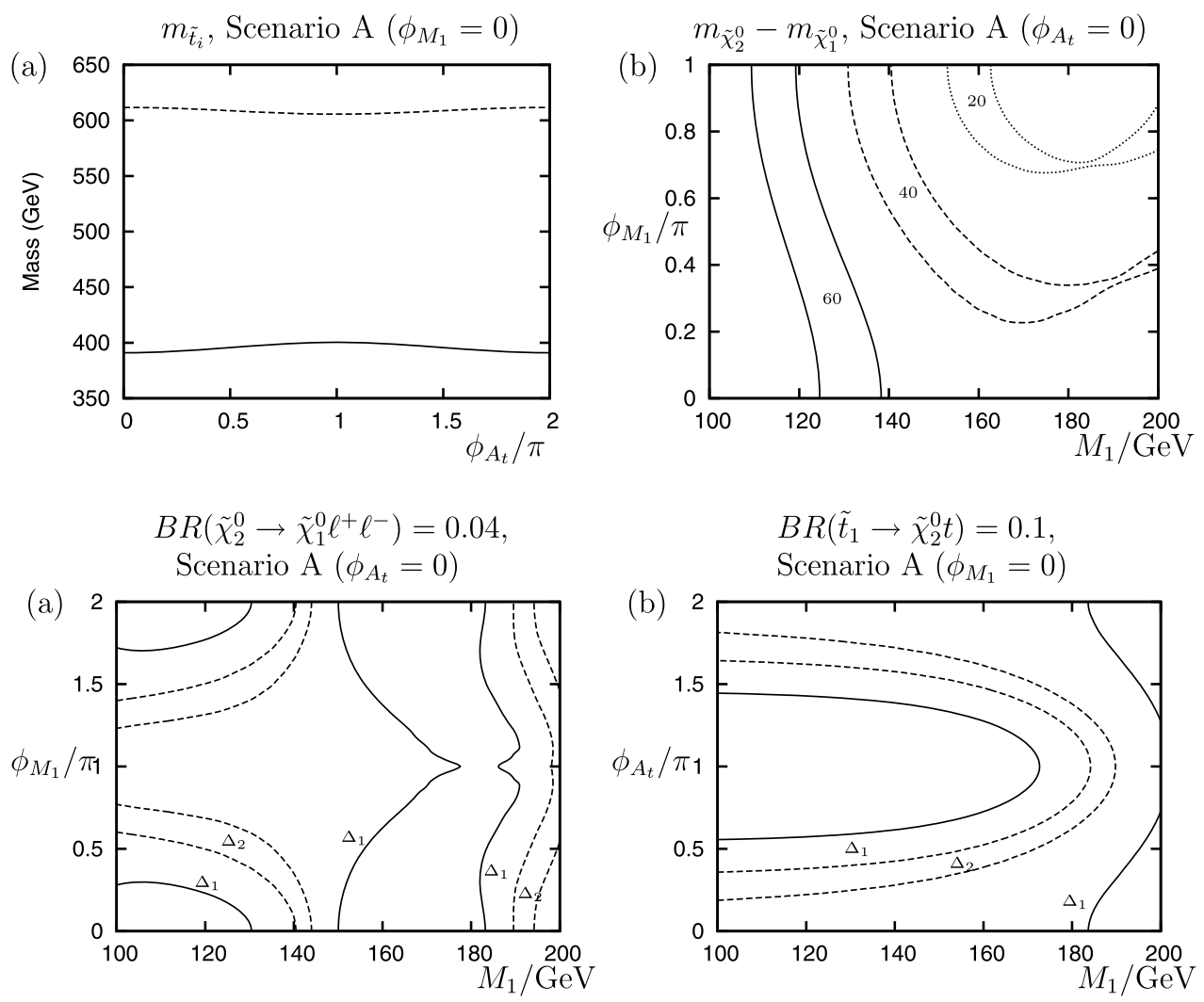


\section{Conclusions}

We have studied direct stop production followed by the decay $\tilde{t}_{1} \rightarrow t \tilde{\chi}_{2}^{0}, \tilde{\chi}_{2}^{0} \rightarrow \ell^{+} \ell^{-} \tilde{\chi}_{1}^{0}$, where the latter is a threebody decay and provide compact analytical expressions for the amplitude and phase space. We have specifically concentrated on measuring the CP-violating phases of the parameters $M_{1}$ and $A_{t}$.

We have provided a thorough analysis of the contributions to this process which lead to non-zero asymmetries in the parameters $\mathcal{T}_{t}, \mathcal{T}_{b}$ and $\mathcal{T}_{t b}$ formed from triple products of reconstructible final-state particles. These are sensitive to different combinations of the phases mentioned above. We studied three spectra which had different neutralino characteristics at the parton level and also studied the (large) effect of including pdfs which had previously only been roughly estimated in the literature.

We found that with the design integrated luminosity of the LHC of $100 \mathrm{fb}^{-1}$, the statistical errors would probably remain too great to distinguish a non-zero asymmetry measurement from zero for most of the ranges of $\phi_{M_{1}}$ and $\phi_{A_{t}}$, and we recall that this initial study did not include detector or background effects. However, with a luminosity upgrade, the accuracy will improve and it could be possible either to measure a non-zero value or else to provide limits on the possible phases.

Triple products are not the only variables sensitive to the phases of the parameters. We found that a good measurement of the mass difference between the $\tilde{\chi}_{2}^{0}$ and $\tilde{\chi}_{1}^{0}$ neutralinos could constrain significantly the $\left(M_{1}, \phi_{M_{1}}\right)$ parameter space. It is possible that measurements of the two branching ratios $B R\left(\tilde{t}_{1} \rightarrow \tilde{\chi}_{2}^{0} t\right)$ and $B R\left(\tilde{\chi}_{2}^{0} \rightarrow \tilde{\chi}_{1}^{0} \ell^{+} \ell^{-}\right)$could also constrain both $\phi_{M_{1}}$ and $\phi_{A_{t}}$, although this is heavily dependent on the experimental accuracy achieved. However, the disadvantage of both mass differences and branching ratios is that a non-zero value can potentially be faked by other values of the real parameters. This is in contrast to the asymmetries from triple products which are uniquely due to $\mathrm{CP}$ violation. Therefore, even though these will be challenging measurements at the LHC, they are worthwhile experimental objectives.

Acknowledgements We are grateful to Martyn Gigg, David Grellscheid and Peter Richardson for their assistance in the use of Herwig++ for these studies. J.T. thanks Martin White and Dan Tovey for useful discussions. J.M.S. and J.T. are supported by the UK Science and Technology Facilities Council (STFC).

\section{Appendix A: Lagrangian and couplings}

The interaction Lagrangian terms for the production processes are

$\mathcal{L}_{g g g}=g_{s} \partial^{\nu} G_{\mu}^{a} g^{\mu \rho} f_{a b c} G_{\nu}^{b} G_{\rho}^{c}$,
$\mathcal{L}_{\tilde{q} \tilde{q} g}=i g_{s} T_{r s}^{a} \delta_{i j} G_{\mu}^{a} \tilde{q}_{j r}^{*} \stackrel{\leftrightarrow}{\partial^{\mu}} \tilde{q}_{i s}$,

$\mathcal{L}_{\tilde{q} \tilde{q} g g}=\frac{1}{2} g_{s}^{2}\left(\frac{1}{3} \delta_{a b}+d_{a b c} T^{c}\right) G_{\mu}^{a} G^{b \mu} \tilde{q}_{j}^{*} \tilde{q}_{i}$.

The interaction Lagrangian terms for $\tilde{\chi}_{2}^{0}$ decay are

$\mathcal{L}_{Z^{0} \ell^{+} \ell^{-}}=-\frac{g}{\cos \Theta_{W}} Z_{\mu} \bar{\ell} \gamma^{\mu}\left[L_{\ell} P_{L}+R_{\ell} P_{R}\right] \ell$,

$\mathcal{L}_{Z^{0} \tilde{\chi}_{m}^{0} \tilde{\chi}_{n}^{0}}=\frac{1}{2} \frac{g}{\cos \Theta_{W}} Z_{\mu} \overline{\tilde{\chi}}_{m}^{0} \gamma^{\mu}\left[O_{m n}^{\prime \prime L} P_{L}+O_{m n}^{\prime \prime R} P_{R}\right] \tilde{\chi}_{n}^{0}$,

$\mathcal{L}_{\ell \tilde{\ell} \tilde{\chi}_{k}^{0}}=g \bar{\ell}\left(a_{j k}^{\tilde{\ell}} P_{R}+b_{j k}^{\tilde{\ell}} P_{L}\right) \tilde{\chi}_{k}^{0} \ell_{j}+$ h.c.,

where the couplings $a_{j k}^{\tilde{\ell}}$ and $b_{j k}^{\tilde{\ell}}$ are given by

$a_{i k}^{\tilde{\ell}}=\sum_{n=1}^{2}\left(\mathcal{R}_{i n}^{\tilde{\ell}}\right)^{*} \mathcal{A}_{k n}^{\ell}, \quad b_{i k}^{\tilde{\ell}}=\sum_{n=1}^{2}\left(\mathcal{R}_{i n}^{\tilde{\ell}}\right)^{*} \mathcal{B}_{k n}^{\ell}$.

Here $\mathcal{R}_{i n}^{\tilde{\ell}}$ is the mixing matrix of the squarks and

$\mathcal{A}_{k}^{\ell}=\left(\begin{array}{c}f_{L k}^{\ell} \\ h_{R k}^{\ell}\end{array}\right), \quad \mathcal{B}_{k}^{\ell}=\left(\begin{array}{c}h_{L k}^{\ell} \\ f_{R k}^{\ell}\end{array}\right)$,

with

$f_{L k}^{\ell}=-\sqrt{2} e_{\ell} \sin \theta_{W} N_{k 1}-\sqrt{2}\left(T_{3 \ell}-e_{\ell} \sin ^{2} \theta_{W}\right) \frac{N_{k 2}}{\cos \theta_{W}}$,

$f_{R k}^{\ell}=-\sqrt{2} e_{\ell} \sin \theta_{W}\left(\tan \theta_{W} N_{k 2}^{*}-N_{k 1}^{*}\right)$,

$h_{L k}^{\ell}=-Y_{\ell}\left(N_{k 3}^{*} \sin \beta-N_{k 4}^{*} \cos \beta\right)$

$=\left(h_{R k}^{\ell}\right)^{*}$,

$O_{m n}^{\prime \prime L}=-\frac{1}{2}\left(N_{m 3} N_{n 3}^{*}-N_{m 4} N_{n 4}^{*}\right) \cos 2 \beta$

$-\frac{1}{2}\left(N_{m 3} N_{n 4}^{*}+N_{m 4} N_{n 3}^{*}\right) \sin 2 \beta$,

$O_{m n}^{\prime \prime R}=-O_{m n}^{\prime \prime L *}$,

$L_{\ell}=T_{3 \ell}-e_{\ell} \sin ^{2} \Theta_{W}, \quad R_{\ell}=-e_{\ell} \sin ^{2} \Theta_{W}$,

where $P_{L, R}=\frac{1}{2}\left(1 \mp \gamma_{5}\right), Y_{t}=m_{t} /\left(\sqrt{2} m_{W} \sin \beta\right)$. Here, $g$ is the weak coupling constant $\left(g=e / \cos \Theta_{W}, e>0\right), e_{\ell}$ and $T_{3 \ell}$ are the charge (in units of $e$ ) and the third component of the weak isospin of the fermion $\ell, \Theta_{W}$ is the weak mixing angle and $\tan \beta=v_{2} / v_{1}$ is the ratio of the vacuum expectation values of the Higgs fields. The unitary $(4 \times 4)$ matrix $N_{m k}$ that diagonalises the complex symmetric neutralino mass matrix is given in the basis $\left(\tilde{\gamma}, \tilde{Z}^{0}, \tilde{H}_{1}^{0}, \tilde{H}_{2}^{0}\right)$ by [55]: 
$N=\left(\begin{array}{cccc}M_{1} e^{i \phi_{M_{1}}} c_{W}^{2}+M_{2} s_{W}^{2} & \left(M_{1} e^{i \phi_{M_{1}}}-M_{2}\right) s_{W} c_{W} & 0 & 0 \\ \left(M_{1} e^{i \phi_{M_{1}}}-M_{2}\right) s_{W} c_{W} & M_{1} e^{i \phi_{M_{1}}} c_{W}^{2}+M_{2} s_{W}^{2} & M_{Z} & 0 \\ 0 & M_{Z} & \mu e^{i \phi_{\mu}} s_{2 \beta} & -\mu e^{i \phi_{\mu}} c_{2 \beta} \\ 0 & 0 & -\mu e^{i \phi_{\mu}} c_{2 \beta} & -\mu e^{i \phi_{\mu}} s_{2 \beta}\end{array}\right)$,

where the abbreviations $s_{W}=\sin \theta_{W}, c_{W}=\cos \theta_{W}, s_{2 \beta}=$ $\sin 2 \beta, c_{2 \beta}=\cos 2 \beta$ have been used.

The interaction Lagrangian for $\tilde{t}$ decay is

$\mathcal{L}_{q \tilde{q} \tilde{\chi}_{k}^{0}}=g \bar{q}\left(a_{i k}^{\tilde{q}} P_{R}+b_{i k}^{\tilde{q}} P_{L}\right) \tilde{\chi}_{k}^{0} \tilde{q}_{i}+$ h.c.,

where the couplings $a_{i k}^{\tilde{q}}$ and $b_{i k}^{\tilde{q}}$ are given by

$a_{i k}^{\tilde{q}}=\sum_{n=1}^{2}\left(\mathcal{R}_{i n}^{\tilde{q}}\right)^{*} \mathcal{A}_{k n}^{q}, \quad b_{i k}^{\tilde{q}}=\sum_{n=1}^{2}\left(\mathcal{R}_{i n}^{\tilde{q}}\right)^{*} \mathcal{B}_{k n}^{q}$.

Here $\mathcal{R}_{i n}^{\tilde{q}}$ is the mixing matrix of the squarks and

$\mathcal{A}_{k}^{q}=\left(\begin{array}{c}f_{L k}^{q} \\ h_{R k}^{q}\end{array}\right), \quad \mathcal{B}_{k}^{q}=\left(\begin{array}{c}h_{L k}^{q} \\ f_{R k}^{q}\end{array}\right)$,

with

$f_{L k}^{q}=-\sqrt{2} e_{q} \sin \theta_{W} N_{k 1}-\sqrt{2}\left(T_{3 q}-e_{q} \sin ^{2} \theta_{W}\right) \frac{N_{k 2}}{\cos \theta_{W}}$,

$f_{R k}^{q}=-\sqrt{2} e_{q} \sin \theta_{W}\left(\tan \theta_{W} N_{k 2}^{*}-N_{k 1}^{*}\right)$,

$h_{L k}^{t}=Y_{t}\left(N_{k 3}^{*} \sin \beta-N_{k 4}^{*} \cos \beta\right)$

$$
=\left(h_{R k}^{t}\right)^{*} \text {. }
$$

We also use the following relations from [21]. The left-right mixing of the stop squarks is described by a hermitian $2 \times 2$ mass matrix which reads as follows in the basis $\left(\tilde{t}_{L}, \tilde{t}_{R}\right)$ :

$\mathcal{L}_{M}^{\tilde{t}}=-\left(\tilde{t}_{L}^{\dagger}, \tilde{t}_{R}^{\dagger}\right)\left(\begin{array}{cc}M_{\tilde{t}_{L L}}^{2} & e^{-i \phi_{\tilde{t}}}\left|M_{\tilde{t}_{L R}}^{2}\right| \\ e^{i \phi_{\tilde{t}}}\left|M_{\tilde{t}_{L R}}^{2}\right| & M_{\tilde{t}_{R R}}^{2}\end{array}\right)\left(\begin{array}{c}\tilde{t}_{L} \\ \tilde{t}_{R}\end{array}\right)$,

where

$M_{\tilde{t}_{L L}}^{2}=M_{\tilde{Q}}^{2}+\left(\frac{1}{2}-\frac{2}{3} \sin ^{2} \Theta_{W}\right) \cos 2 \beta m_{Z}^{2}+m_{t}^{2}$,

$M_{\tilde{t}_{R R}}^{2}=M_{\tilde{U}}^{2}+\frac{2}{3} \sin ^{2} \Theta_{W} \cos 2 \beta m_{Z}^{2}+m_{t}^{2}$,

$M_{\tilde{t}_{R L}}^{2}=\left(M_{\tilde{t}_{L R}}^{2}\right)^{*}=m_{t}\left(A_{t}-\mu^{*} \cot \beta\right)$,

$\phi_{\tilde{t}}=\arg \left[A_{t}-\mu^{*} \cot \beta\right]$.

Here $\tan \beta=v_{2} / v_{1}$ with $v_{1}\left(v_{2}\right)$ being the vacuum expectation value of the Higgs field $H_{1}^{0}\left(H_{2}^{0}\right), m_{t}$ is the mass of the top quark and $\Theta_{W}$ is the weak mixing angle, $\mu$ is the Higgs-higgsino mass parameter and $M_{\tilde{Q}}, M_{\tilde{U}}, A_{t}$ are the soft SUSY-breaking parameters of the stop squark system. The mass eigenstates $\tilde{t}_{i}$ are $\left(\tilde{t}_{1}, \tilde{t}_{2}\right)=\left(\tilde{t}_{L}, \tilde{t}_{R}\right) \mathcal{R}^{\tilde{t}^{T}}$ with

$\mathcal{R}^{\tilde{t}}=\left(\begin{array}{cc}e^{i \phi_{\tilde{t}}} \cos \theta_{\tilde{t}} & \sin \theta_{\tilde{t}} \\ -\sin \theta_{\tilde{t}} & e^{-i \phi_{\tilde{t}}} \cos \theta_{\tilde{t}}\end{array}\right)$,

where

$\cos \theta_{\tilde{t}}=\frac{-\left|M_{\tilde{t}_{L R}}^{2}\right|}{\sqrt{\left|M_{\tilde{t}_{L R}}^{2}\right|^{2}+\left(m_{\tilde{t}_{1}}^{2}-M_{\tilde{t}_{L L}}^{2}\right)^{2}}}$,

$\sin \theta_{\tilde{t}}=\frac{M_{\tilde{t}_{L L}}^{2}-m_{\tilde{t}_{1}}^{2}}{\sqrt{\left|M_{\tilde{t}_{L R}}^{2}\right|^{2}+\left(m_{\tilde{t}_{1}}^{2}-M_{\tilde{t}_{L L}}^{2}\right)^{2}}}$.

The mass eigenvalues are

$$
\begin{aligned}
m_{\tilde{t}_{1,2}}^{2}= & \frac{1}{2}\left(\left(M_{\tilde{t}_{L L}}^{2}+M_{\tilde{t}_{R R}}^{2}\right)\right. \\
& \left.\mp \sqrt{\left(M_{\tilde{t}_{L L}}^{2}-M_{\tilde{t}_{R R}}^{2}\right)^{2}+4\left|M_{\tilde{t}_{L R}}^{2}\right|^{2}}\right) .
\end{aligned}
$$

We note that we have $\phi_{\tilde{t}} \approx \phi_{A_{t}}$ for $\left|A_{t}\right| \gg|\mu| \cot \beta$.

\section{Appendix B: Explicit expressions for the squared amplitude}

B.1 Neutralino production $\tilde{t}_{i} \rightarrow \tilde{\chi}_{j}^{0}$

Here we give the analytic expression for the production density matrix:

$$
\left|M\left(\tilde{t}_{i} \rightarrow \tilde{\chi}_{j}^{0} t\right)\right|^{2}=P\left(\tilde{\chi}_{j}^{0} t\right)+\Sigma_{P}^{a}\left(\tilde{\chi}_{j}^{0}\right)+\Sigma_{P}^{b}(t)+\Sigma_{P}^{a b}\left(\tilde{\chi}_{j}^{0} t\right),
$$

whose spin-independent contribution reads

$$
\begin{aligned}
P\left(\tilde{\chi}_{j}^{0} t\right)= & \frac{g^{2}}{2}\left\{\left(\left|a_{i j}\right|^{2}+\left|b_{i j}\right|^{2}\right)\left(p_{t} p_{\tilde{\chi}_{j}^{0}}\right)\right. \\
& \left.-2 m_{t} m_{\tilde{\chi}_{j}^{0}} \operatorname{Re}\left(a_{i j} b_{i j}^{*}\right)\right\},
\end{aligned}
$$


where $p_{t}$ and $p_{\tilde{\chi}_{k}^{0}}$ denote the four-momenta of the $t$-quark and the neutralino $\tilde{\chi}_{k}^{0}$. The coupling constants can be simplified and shown to be

$$
\begin{aligned}
& \left(\left|a_{i j}\right|^{2}+\left|b_{i j}\right|^{2}\right) \\
& =\left|h_{R j}^{t}\right|^{2}+\cos ^{2} \theta_{\tilde{t}}\left|f_{L j}^{t}\right|^{2}+\sin ^{2} \theta_{\tilde{t}}\left|f_{R j}^{t}\right|^{2} \\
& \quad+2 \sin \theta_{\tilde{t}} \cos \theta_{\tilde{t}} \operatorname{Re}\left[e^{-i \phi_{\tilde{t}}} h_{R j}^{t *}\left(f_{R j}^{t}+f_{L j}^{t}\right)\right],
\end{aligned}
$$

$$
\begin{aligned}
\operatorname{Re}\left(a_{i j} b_{i j}^{*}\right)= & \cos ^{2} \theta_{\tilde{t}} \operatorname{Re}\left(f_{R j}^{t} h_{R j}^{t}\right)+\sin ^{2} \theta_{\tilde{t}} \operatorname{Re}\left(f_{R j}^{t *} h_{R j}^{t}\right) \\
& +\frac{1}{2} \sin 2 \theta_{\hat{t}} \operatorname{Re}\left[e^{i \phi_{\tilde{t}}}\left|h_{R j}^{t}\right|^{2}+e^{-i \phi_{\tilde{t}}} f_{L j}^{t} f_{R j}^{t *}\right],
\end{aligned}
$$

where $\phi_{\tilde{t}}$ is given in (A.26).

The spin-dependent contributions are T-even and are given by

$\Sigma_{P}^{a}\left(\tilde{\chi}_{2}^{0}\right)=\frac{g^{2}}{2}\left\{\left(\left|b_{i j}\right|^{2}-\left|a_{i j}\right|^{2}\right) m_{\tilde{\chi}_{j}^{0}}\left(p_{t} s^{a}\left(\tilde{\chi}_{j}^{0}\right)\right)\right\}$,

$\Sigma_{P}^{b}(t)=\frac{g^{2}}{2}\left\{\left(\left|b_{i j}\right|^{2}-\left|a_{i j}\right|^{2}\right) m_{t}\left(p_{\tilde{\chi}_{j}^{0}} s^{b}(t)\right)\right\}$,

where $s^{a}\left(\tilde{\chi}_{j}^{0}\right)\left(s^{b}(t)\right)$ denote the spin-basis vectors of the neutralino $\tilde{\chi}_{j}^{0}$ (t-quark). Again the coupling constant can be simplified as

$$
\begin{aligned}
\left(\left|b_{i j}\right|^{2}-\left|a_{i j}\right|^{2}\right) & \\
= & \cos 2 \theta_{\tilde{t}}\left|h_{R j}^{t}\right|^{2}-\cos ^{2} \theta_{\tilde{t}}\left|f_{L j}^{t}\right|^{2}+\sin ^{2} \theta_{\tilde{t}}\left|f_{R j}^{t}\right|^{2} \\
& -2 \sin \theta_{\tilde{t}} \cos \theta_{\tilde{t}} \operatorname{Re}\left[e^{-i \phi_{\tilde{t}}} h_{R j}^{t *}\left(f_{R j}^{t}+f_{L j}^{t}\right)\right] .
\end{aligned}
$$

The three spin-basis four-vectors $s^{1}, s^{2}$ and $s^{3}$ form a right-handed system and provide, together with the momentum, an orthogonal basis system. They are chosen as:

$s^{1}\left(\tilde{\chi}_{j}^{0}\right)=\left(0, \frac{\left(\vec{p}_{\tilde{\chi}_{j}^{0}} \times \vec{p}_{\tilde{t}_{i}}\right) \times \vec{p}_{\tilde{\chi}_{j}^{0}}}{\left|\left(\vec{p}_{\tilde{\chi}_{j}^{0}} \times \vec{p}_{\tilde{t}_{i}}\right) \times \vec{p}_{\tilde{\chi}_{j}^{0}}\right|}\right)$,

$s^{2}\left(\tilde{\chi}_{j}^{0}\right)=\left(0, \frac{\vec{p}_{\tilde{\chi}_{j}^{0}} \times \vec{p}_{\tilde{t}_{i}}}{\left|\vec{p}_{\tilde{\chi}_{j}^{0}} \times \vec{p}_{\tilde{t}_{i}}\right|}\right)$,

$s^{3}\left(\tilde{\chi}_{j}^{0}\right)=\frac{1}{m_{\tilde{\chi}_{j}^{0}}}\left(\left|\vec{p}_{\tilde{\chi}_{j}^{0}}\right|, \frac{E_{\tilde{\chi}_{j}^{0}}}{\left|\vec{p}_{\tilde{\chi}_{j}^{0}}\right|} \vec{p}_{\tilde{\chi}_{j}^{0}}\right)$.

The spin system for the top quark has been chosen analogously:

$s^{1}(t)=\left(0, \frac{\left(\vec{p}_{t} \times \vec{p}_{\tilde{\chi}_{j}^{0}}\right) \times \vec{p}_{t}}{\left|\left(\vec{p}_{t} \times \vec{p}_{\tilde{\chi}_{j}^{0}}\right) \times \vec{p}_{t}\right|}\right)$, $s^{2}(t)=\left(0, \frac{\vec{p}_{t} \times \vec{p}_{\tilde{\chi}_{j}^{0}}}{\left|\vec{p}_{t} \times \vec{p}_{\tilde{\chi}_{j}^{0}}\right|}\right)$,

$s^{3}(t)=\frac{1}{m_{t}}\left(\left|\vec{p}_{t}\right|, \frac{E_{t}}{\left|\vec{p}_{t}\right|} \vec{p}_{t}\right)$,

and $E_{t}$ and $E_{\tilde{\chi}_{j}^{0}}$ denote the energies of the top quark and the neutralino $\tilde{\chi}_{j}^{0}$, respectively.

The terms that depend simultaneously on the spin of the top quark and of the neutralino can be split into T-even, $\Sigma_{P}^{a b, \mathrm{E}}\left(\tilde{\chi}_{2}^{0} t\right)$, and T-odd, $\Sigma_{P}^{a b, \mathrm{O}}\left(\tilde{\chi}_{2}^{0} t\right)$, contributions:

$$
\begin{aligned}
\Sigma_{P}^{a b, \mathrm{E}}\left(\tilde{\chi}_{2}^{0} t\right)= & \frac{g^{2}}{2}\left\{2 \operatorname { R e } ( a _ { i j } b _ { i j } ^ { * } ) \left[\left(s^{a}\left(\tilde{\chi}_{j}^{0}\right) p_{t}\right)\left(s^{b}(t) p_{\tilde{\chi}_{j}^{0}}\right)\right.\right. \\
& \left.-\left(p_{t} p_{\tilde{\chi}_{j}^{0}}\right)\left(s^{a}\left(\tilde{\chi}_{j}^{0}\right) s^{b}(t)\right)\right] \\
& \left.+m_{t} m_{\tilde{\chi}_{j}^{0}}\left(s^{a}\left(\tilde{\chi}_{j}^{0}\right) s^{b}(t)\right)\left(\left|a_{i j}\right|^{2}+\left|b_{i j}\right|^{2}\right)\right\},
\end{aligned}
$$

$\Sigma_{P}^{a b, \mathrm{O}}\left(\tilde{\chi}_{2}^{0} t\right)=-g^{2} \operatorname{Im}\left(a_{i j} b_{i j}^{*}\right) f_{4}^{a b}$,

where the T-odd kinematical factor is given by

$f_{4}^{a b}=\epsilon_{\mu \nu \rho \sigma} s^{a, \mu}\left(\tilde{\chi}_{j}^{0}\right) p_{\tilde{\chi}_{j}^{0}}^{v} s^{b, \rho}(t) p_{t}^{\sigma}$,

and the coupling constant by

$$
\begin{aligned}
\operatorname{Im}\left(a_{i j} b_{i j}^{*}\right)= & \cos ^{2} \theta_{\tilde{t}} \operatorname{Im}\left(f_{R j}^{t} h_{R j}^{t}\right)+\sin ^{2} \theta_{\tilde{t}} \operatorname{Im}\left(f_{R j}^{t *} h_{R j}^{t}\right) \\
& +\frac{1}{2} \sin 2 \theta_{\tilde{t}} \operatorname{Im}\left[e^{i \phi_{\tilde{t}}}\left|h_{R j}^{t}\right|^{2}\right. \\
& \left.+e^{-i \phi_{\tilde{t}}} f_{L j}^{t} f_{R j}^{t *}\right] .
\end{aligned}
$$

B.2 Neutralino three-body decay $\tilde{\chi}_{j}^{0} \rightarrow \tilde{\chi}_{k}^{0} \ell^{+} \ell^{-}$

Here we give the analytical expressions for the different contributions to the decay density matrix for the three-body decay, where we sum over the spins of the final-state particles [39]. The contributions independent of the polarisation of the neutralino $\tilde{\chi}_{j}^{0}$

$$
\begin{aligned}
D\left(\tilde{\chi}_{j}^{0}\right)= & D(Z Z)+D\left(Z \tilde{\ell}_{L}\right)+D\left(Z \tilde{\ell}_{R}\right)+D\left(\tilde{\ell}_{L} \tilde{\ell}_{L}\right) \\
& +D\left(\tilde{\ell}_{R} \tilde{\ell}_{R}\right),
\end{aligned}
$$

are given by

$$
\begin{aligned}
D(Z Z)= & 8 \frac{g^{4}}{\cos ^{4} \Theta_{W}}|\Delta(Z)|^{2}\left(L_{\ell}^{2}+R_{\ell}^{2}\right) \\
& \times\left[\left|O_{k j}^{\prime \prime}\right|^{2}\left(g_{1}+g_{2}\right)+\left(\operatorname{Re} O_{k j}^{\prime \prime}\right)^{2}\right. \\
& \left.-\left\langle\left(\operatorname{Im} O_{k j}^{\prime \prime} L\right)^{2}\right) g_{3} !\right],
\end{aligned}
$$




$$
\begin{aligned}
D\left(Z \tilde{\ell}_{L}\right)= & 4 \frac{g^{4}}{\cos ^{2} \Theta_{W}} L_{\ell} \operatorname{Re}\left\{\Delta ( Z ) \left[f_{\ell j}^{L} f_{\ell k}^{L *} \Delta^{t *}\left(\tilde{\ell}_{L}\right)\right.\right. \\
& \times\left(2 O_{k j}^{\prime \prime} L g_{1}+O_{k j}^{\prime \prime} g_{3}\right) \\
& \left.\left.+f_{\ell j}^{L *} f_{\ell k}^{L} \Delta^{u *}\left(\tilde{\ell}_{L}\right)\left(2 O_{k j}^{\prime \prime} L * g_{2}+O_{k j}^{\prime \prime} g_{3}\right)\right]\right\},
\end{aligned}
$$

$$
\begin{aligned}
D\left(\tilde{\ell}_{L} \tilde{\ell}_{L}\right)= & 2 g^{4}\left[\left|f_{\ell j}^{L}\right|^{2}\left|f_{\ell k}^{L}\right|^{2}\left(\left|\Delta^{t}\left(\tilde{\ell}_{L}\right)\right|^{2} g_{1}+\left|\Delta^{u}\left(\tilde{\ell}_{L}\right)\right|^{2} g_{2}\right)\right. \\
& \left.+\operatorname{Re}\left\{\left(f_{\ell j}^{L *}\right)^{2}\left(f_{\ell k}^{L}\right)^{2} \Delta^{t}\left(\tilde{\ell}_{L}\right) \Delta^{u *}\left(\tilde{\ell}_{L}\right)\right\} g_{3}\right],
\end{aligned}
$$

where $\Delta(Z)$ and $\Delta^{t, u}\left(\tilde{\ell}_{L}\right)$ denote the propagators of the virtual particles in the direct channel and in both crossed channels (labelled $t, u$, cf. Fig. 2.2).

The quantities $D\left(Z \tilde{\ell}_{R}\right), D\left(\tilde{\ell}_{R} \tilde{e}_{R}\right)$ can be derived from (B.20), (B.21) by the substitutions

$$
\begin{aligned}
L_{\ell} \rightarrow R_{\ell}, & \Delta^{t, u}\left(\tilde{\ell}_{L}\right) \rightarrow \Delta^{t, u}\left(\tilde{\ell}_{R}\right), \\
O_{k j}^{\prime \prime L} \rightarrow O_{k j}^{\prime \prime R}, & f_{\ell j, k}^{L} \rightarrow f_{\ell j, k}^{R} .
\end{aligned}
$$

The kinematical factors are

$$
\begin{aligned}
& g_{1}=\left(p_{\tilde{\chi}_{k}^{0}} p_{\ell^{-}}\right)\left(p_{\tilde{\chi}_{j}^{0}} p_{\ell^{+}}\right), \\
& g_{2}=\left(p_{\tilde{\chi}_{k}^{0}} p_{\ell^{+}}\right)\left(p_{\tilde{\chi}_{j}^{0}} p_{\ell^{-}}\right), \\
& g_{3}=m_{j} m_{k}\left(p_{\ell^{-}} p_{\ell^{+}}\right) .
\end{aligned}
$$

We can split the terms depending on the polarisation of the neutralino into T-even and T-odd contributions:

$$
\Sigma_{D}^{a}\left(\tilde{\chi}_{j}^{0}\right)=\Sigma_{D}^{a, \mathrm{E}}\left(\tilde{\chi}_{j}^{0}\right)+\Sigma_{D}^{a, \mathrm{O}}\left(\tilde{\chi}_{j}^{0}\right) .
$$

The T-even contributions depending on the polarisation of the decaying neutralino $\tilde{\chi}_{j}^{0}$

$$
\begin{aligned}
\Sigma_{D}^{a, \mathrm{E}}\left(\tilde{\chi}_{j}^{0}\right)= & \Sigma_{D}^{a, \mathrm{E}}(Z Z)+\Sigma_{D}^{a, \mathrm{E}}\left(Z \tilde{\ell}_{L}\right)+\Sigma_{D}^{a, \mathrm{E}}\left(Z \tilde{\ell}_{R}\right) \\
& +\Sigma_{D}^{a, \mathrm{E}}\left(\tilde{\ell}_{L} \tilde{\ell}_{L}\right)+\Sigma_{D}^{a, \mathrm{E}}\left(\tilde{\ell}_{R} \tilde{\ell}_{R}\right)
\end{aligned}
$$

are

$$
\begin{aligned}
\Sigma_{D}^{a, \mathrm{E}}(Z Z)= & 8 \frac{g^{4}}{\cos ^{4} \Theta_{W}}|\Delta(Z)|^{2}\left(R_{\ell}^{2}-L_{\ell}^{2}\right) \\
& \times\left[-\left[\left(\operatorname{Re} O_{k j}^{\prime \prime} L\right)^{2}-\left(\operatorname{Im} O_{k j}^{\prime \prime} L\right)^{2}\right] g_{3}^{a}\right. \\
& \left.+\left|O_{k j}^{\prime \prime} L\right|^{2}\left(g_{1}^{a}-g_{2}^{a}\right)\right], \\
\Sigma_{D}^{a, \mathrm{E}}\left(Z \tilde{\ell}_{L}\right)= & \frac{4 g^{4}}{\cos ^{2} \Theta_{W}} L_{\ell} \operatorname{Re}\left\{\Delta ( Z ) \left[f_{\ell j}^{L} f_{\ell k}^{L *} \Delta^{t *}\left(\tilde{\ell}_{L}\right)\right.\right. \\
& \times\left(-2 O_{k j}^{\prime \prime} L g_{1}^{a}+O_{k j}^{\prime \prime} L * g_{3}^{a}\right) \\
& \left.\left.+f_{\ell j}^{L *} f_{\ell k}^{L} \Delta^{u *}\left(\tilde{\ell}_{L}\right)\left(2 O_{k j}^{\prime \prime} L * g_{2}^{a}+O_{k j}^{\prime \prime} L g_{3}^{a}\right)\right]\right\},
\end{aligned}
$$

$$
\begin{aligned}
\Sigma_{D}^{a, \mathrm{E}}\left(\tilde{\ell}_{L} \tilde{\ell}_{L}\right)= & 2 g^{4}\left[| f _ { \ell j } ^ { L } | ^ { 2 } | f _ { \ell k } ^ { L } | ^ { 2 } \left[\left|\Delta^{u}\left(\tilde{\ell}_{L}\right)\right|^{2} g_{2}^{a}\right.\right. \\
& \left.-\left|\Delta^{t}\left(\tilde{\ell}_{L}\right)\right|^{2} g_{1}^{a}\right] \\
& \left.+\operatorname{Re}\left\{\left(f_{\ell j}^{L *}\right)^{2}\left(f_{\ell k}^{L}\right)^{2} \Delta^{t}\left(\tilde{\ell}_{L}\right) \Delta^{u *}\left(\tilde{\ell}_{L}\right) g_{3}^{a}\right\}\right],
\end{aligned}
$$

where the contributions $\Sigma_{D}^{a, \mathrm{E}}\left(Z \tilde{\ell}_{R}\right), \Sigma_{D}^{a, \mathrm{E}}\left(\tilde{\ell}_{R} \tilde{\ell}_{R}\right)$ are derived from (B.29), (B.30) by applying the substitutions in (B.22) and in addition $g_{1,2,3}^{a} \rightarrow-g_{1,2,3}^{a}$.

The kinematical factors are

$g_{1}^{a}=m_{j}\left(p_{\tilde{\chi}_{k}^{0}} p_{\ell^{-}}\right)\left(p_{\ell^{+}} s^{a}\right)$,

$g_{2}^{a}=m_{j}\left(p_{\tilde{\chi}_{k}^{0}} p_{\ell^{+}}\right)\left(p_{\ell^{-}} s^{a}\right)$,

$g_{3}^{a}=m_{k}\left[\left(p_{\tilde{\chi}_{j}^{0}} p_{\ell^{+}}\right)\left(p_{\ell^{-}} s^{a}\right)-\left(p_{\tilde{\chi}_{j}^{0}} p_{\ell^{-}}\right)\left(p_{\ell^{+}} s^{a}\right)\right]$.

The T-odd contributions depending on the polarisation of the decaying neutralino $\tilde{\chi}_{j}^{0}$

$$
\begin{aligned}
\Sigma_{D}^{a, \mathrm{O}}\left(\tilde{\chi}_{j}^{0}\right)= & \Sigma_{D}^{a, \mathrm{O}}(Z Z)+\Sigma_{D}^{a, \mathrm{O}}\left(Z \tilde{\ell}_{L}\right)+\Sigma_{D}^{a, \mathrm{O}}\left(Z \tilde{\ell}_{R}\right) \\
& +\Sigma_{D}^{a, \mathrm{O}}\left(\tilde{\ell}_{L} \tilde{\ell}_{L}\right)+\Sigma_{D}^{a, \mathrm{O}}\left(\tilde{\ell}_{R} \tilde{\ell}_{R}\right) .
\end{aligned}
$$

are

$$
\begin{aligned}
\Sigma_{D}^{a, \mathrm{O}}(Z Z)= & 8 \frac{g^{4}}{\cos ^{4} \Theta_{W}}|\Delta(Z)|^{2}\left(L_{\ell}^{2}-R_{\ell}^{2}\right) \\
& \times\left[2 \operatorname{Re}\left(O_{k j}^{\prime \prime} L\right) \operatorname{Im}\left(O_{k j}^{\prime \prime} L\right) i g_{4}^{a}\right], \\
\Sigma_{D}^{a, \mathrm{O}}\left(Z \tilde{\ell}_{L}\right)= & \frac{4 g^{4}}{\cos ^{2} \Theta_{W}} L_{\ell} \\
& \times \operatorname{Re}\left\{\Delta ( Z ) \left[-f_{\ell j}^{L} f_{\ell k}^{L *} O_{k j}^{\prime \prime} L * \Delta^{t *}\left(\tilde{\ell}_{L}\right)\right.\right. \\
& \left.\left.+f_{\ell j}^{L *} f_{\ell k}^{L} O_{k j}^{\prime \prime} L \Delta^{u *}\left(\tilde{\ell}_{L}\right)\right] g_{4}^{a}\right\}, \\
\Sigma_{D}^{a, \mathrm{O}}\left(\tilde{\ell}_{L} \tilde{\ell}_{L}\right)= & 2 g^{4} \operatorname{Re}\left\{\left(f_{\ell j}^{L *}\right)^{2}\left(f_{\ell k}^{L}\right)^{2} \Delta^{t}\left(\tilde{\ell}_{L}\right) \Delta^{u *}\left(\tilde{\ell}_{L}\right) g_{4}^{a}\right\},
\end{aligned}
$$

where the contributions $\Sigma_{D}^{a, \mathrm{O}}\left(Z \tilde{\ell}_{R}\right), \Sigma_{D}^{a, \mathrm{O}}\left(\tilde{\ell}_{R} \tilde{\ell}_{R}\right)$ are derived from (B.29), (B.30) by applying the substitutions in (B.22). The kinematical factor is

$$
g_{4}^{a}=i m_{k} \epsilon_{\mu \nu \rho \sigma} s^{a \mu} p_{\tilde{\chi}_{j}^{0}}^{v} p_{\ell^{-}}^{\rho} p_{\ell^{+}}^{\sigma} .
$$

\section{B.3 Top decay $t \rightarrow W^{+} b$}

We provide analytical expressions for the 2-body decay of the top quark into a $W$-boson and the final-state bottom quark:

$$
D(t)=\frac{g^{2}}{4}\left\{m_{t}^{2}-2 m_{W}^{2}+\frac{m_{t}^{4}}{m_{W}^{2}}\right\} .
$$


The spin-dependent contribution is T-even and reads

$\Sigma_{D}^{b}(t)=-\frac{g^{2}}{2} m_{t}\left\{\left(s^{b}(t) p_{b}\right)+\frac{m_{t}^{2}-m_{W}^{2}}{m_{W}^{2}}\left(s^{b}(t) p_{W}\right)\right\}$

\section{Appendix C: Kinematics}

\section{C.1 Phase space}

The complete cross section for the process can be decomposed into the production cross section and the branching ratios of the subsequent decays:

$$
\begin{aligned}
& d \sigma_{\text {Total }} \\
& =d \sigma\left(g g \rightarrow \tilde{t}_{1} \overline{\tilde{t}_{1}}\right) \frac{E_{\tilde{t}_{1}}}{m_{\tilde{t}_{1}} \Gamma_{\tilde{t}_{1}}} d \Gamma\left(\tilde{t}_{1} \rightarrow t \tilde{\chi}_{2}^{0}\right) \\
& \quad \times \frac{E_{\tilde{\chi}_{2}^{0}}}{m_{\tilde{\chi}_{2}^{0}} \Gamma_{\tilde{\chi}_{2}^{0}}} d \Gamma\left(\tilde{\chi}_{2}^{0} \rightarrow \tilde{\chi}_{1}^{0} l^{+} l^{-}\right) \frac{E_{t}}{m_{t} \Gamma_{t}} d \Gamma\left(t \rightarrow W^{+} b\right),
\end{aligned}
$$

where the factors $E / m \Gamma$ come from the use of the narrowwidth approximation for the propagators of the $\tilde{t}, \tilde{\chi}_{2}^{0}$ and $t$. This approximation is valid for $(\Gamma / m)^{2} \ll 1$, which is satisfied for $\Gamma_{t} \sim 1.5 \mathrm{GeV}$ [43] and $\Gamma_{\tilde{t}} \sim 4 \mathrm{GeV}$. It is also trivially satisfied in the case of $\Gamma_{\tilde{\chi}_{2}^{0}} \sim 10^{-4}$ where the width is small because only the three-body decay is kinematically possible.
We have:

$$
\begin{aligned}
& d \Gamma\left(\tilde{t}_{1} \rightarrow t \tilde{\chi}_{2}^{0}\right)=\frac{2}{E_{\tilde{t}_{1}}} P\left(\tilde{\chi}_{2}^{0} t\right) d \Phi_{\tilde{t}}, \\
& d \Gamma\left(\tilde{\chi}_{2}^{0} \rightarrow \tilde{\chi}_{1}^{0} l^{+} l^{-}\right)=\frac{1}{4 E_{\tilde{\chi}_{2}}} D\left(\tilde{\chi}_{2}^{0}\right) d \Phi_{\tilde{\chi}_{2}^{0}}, \\
& d \Gamma\left(t \rightarrow W^{+} b\right)=\frac{1}{4 E_{t}} D(t) d \Phi_{t},
\end{aligned}
$$

where the phase-space factors in the laboratory system are given by

$$
\begin{aligned}
& d \Phi_{\tilde{t}}=\frac{1}{(2 \pi)^{2}} \frac{\left|\mathbf{p}_{\tilde{\chi}_{2}^{0}}^{ \pm}\right|^{2}}{2\left|E_{\tilde{t}}\right| \mathbf{p}_{\tilde{\chi}_{2}^{0}}^{ \pm}\left|-E_{\tilde{\chi}_{2}^{0}}^{ \pm}\right| \mathbf{p}_{\tilde{t}}\left|\cos \theta_{\tilde{t}}\right|} d \Omega_{\tilde{t}} \\
& d \Phi_{\tilde{\chi}_{2}^{0}}=\frac{1}{8(2 \pi)^{5}} \frac{E_{l^{+}}}{|| \mathbf{p}_{\tilde{\chi}_{2}^{0}}\left|\cos \theta_{l^{+}}-E_{\tilde{\chi}_{1}^{0}}-E_{l^{+}}-E_{l^{-}} \cos \alpha\right|} \\
& E_{l^{-}} d E_{l^{-}} d \Omega_{l^{+}} d \Omega_{l^{-}}, \\
& d \Phi_{t}=\frac{1}{(2 \pi)^{2}} \frac{E_{b}}{2|| \mathbf{p}_{t}\left|\cos \theta_{b}-E_{W}-E_{b}\right|} d \Omega_{b}
\end{aligned}
$$

There is a subtlety in the phase-space calculation, namely that there can be two solutions for $\mathbf{p}_{\tilde{\chi}_{2}^{0}}$. If $\left|\mathbf{p}_{\tilde{\chi}_{2}^{0}}\right|<p_{0}$ where $p_{0}=\lambda^{\frac{1}{2}}\left(m_{\tilde{t}}^{2}, m_{\tilde{\chi}_{2}^{0}}^{2}, m_{t}^{2}\right) / 2 m_{\tilde{\chi}_{2}^{0}}$, then the decay angle, $\theta_{\tilde{t}}=\measuredangle\left(\mathbf{p}_{\tilde{t}}, \mathbf{p}_{t}\right)$, is unconstrained and there is only one solution. However, if $\mathbf{p}_{\tilde{\chi}_{2}^{0}}>p_{0}$, then the angle is constrained by $\sin \theta_{\tilde{t}}^{\max }=p_{0} /\left|\mathbf{p}_{\tilde{\chi}_{2}^{0}}\right|$ and there are two physical solutions

$\left|\mathbf{p}_{\tilde{\chi}_{2}^{0}}\right|=\frac{\left(m_{\tilde{t}}^{2}+m_{\tilde{\chi}_{2}^{0}}^{2}-m_{\theta}^{2}\right)\left|\mathbf{p}_{\tilde{t}}\right| \cos \theta_{\tilde{t}} \pm E_{\tilde{t}} \sqrt{\lambda\left(m_{\tilde{t}}^{2}, m_{\tilde{\chi}_{2}^{0}}^{2}, m_{t}^{2}\right)-4\left|\mathbf{p}_{\tilde{t}}\right|^{2} m_{\tilde{\chi}_{2}^{0}}^{2}\left(1-\cos ^{2} \theta_{\tilde{t}}\right)}}{2\left|\mathbf{p}_{\tilde{t}}\right|^{2}\left(1-\cos ^{2} \theta_{\tilde{t}}\right)+2 m_{\tilde{t}}^{2}}$.

For the region of phase space where two solutions exist the cross section becomes a summation of the solutions for each of the subsequent decay chains.

\section{C.2 Integration limits}

When evaluating the phase-space integral at the parton level, kinematical limits need to be determined on some of the variables and these are listed below.

If $\left|\mathbf{p}_{\tilde{\chi}_{2}^{0}}\right|<p_{0}$, where $p_{0}=\lambda^{\frac{1}{2}}\left(m_{\tilde{t}}^{2}, m_{\tilde{\chi}_{2}^{0}}^{2}, m_{t}^{2}\right) / 2 m_{\tilde{\chi}_{2}^{0}}$, there are two solutions for $\mathbf{p}_{\tilde{\chi}_{2}^{0}},($ C.8), and the decay angle of the $\tilde{t}$ is constrained by

$\sin \theta_{\tilde{t}}<\frac{\lambda^{\frac{1}{2}}\left(m_{\tilde{t}}^{2}, m_{\tilde{\chi}_{2}^{0}}^{2}, m_{t}^{2}\right)}{2\left|\mathbf{p}_{\tilde{t}}\right| m_{\tilde{\chi}_{2}^{0}}}$.

The three-body-decay phase space of the $\tilde{\chi}_{2}^{0}$ also has limits:

$E_{\ell^{-}}<\frac{m_{\tilde{\chi}_{2}^{0}}-m_{\tilde{\chi}_{1}^{0}}}{2\left(E_{\tilde{\chi}_{2}^{0}}-\left|p_{\tilde{\chi}_{2}^{0}}\right|\right)}$

$\cos \theta_{\ell^{-}}<\frac{2 E_{\tilde{\chi}_{2}^{0}} E_{\ell^{-}}+m_{\tilde{\chi}_{1}^{0}}-m_{\tilde{\chi}_{2}^{0}}}{2 E_{\ell^{-}}\left|p_{\tilde{\chi}_{2}^{0}}\right|}$ 


\section{References}

1. O. Buchmueller et al., Prediction for the lightest higgs boson mass in the CMSSM using indirect experimental constraints. Phys. Lett. B 657, 87-94 (2007). arXiv:0707.3447 [hep-ph]

2. S. Heinemeyer, X. Miao, S. Su, G. Weiglein, B-physics observables and electroweak precision data in the CMSSM, mGMSB and mAMSB. arXiv:0805.2359 [hep-ph]

3. S. Hesselbach, Effects of CP-violating phases in supersymmetry. Acta Phys. Polon. B 35, 2739-2751 (2004). arXiv:hep-ph/ 0410174

4. S. Hesselbach, CP violation in SUSY particle production and decay. arXiv:0709.2679 [hep-ph]

5. J.A. Williams, CP violation in charged Higgs production. arXiv: hep-ph/0505121

6. P. Langacker, G. Paz, L.-T. Wang, I. Yavin, A T-odd observable sensitive to CP violating phases in squark decay. J. High Energy Phys. 07, 055 (2007). arXiv:hep-ph/0702068

7. T. Ibrahim, P. Nath, The neutron and the electron electric dipole moment in $N=1$ supergravity unification. Phys. Rev. D 57, 478488 (1998). arXiv:hep-ph/9708456

8. M. Brhlik, G.J. Good, G.L. Kane, Electric dipole moments do not require the CP-violating phases of supersymmetry to be small. Phys. Rev. D 59, 115004 (1999). arXiv:hep-ph/9810457

9. A. Bartl, T. Gajdosik, W. Porod, P. Stockinger, H. Stremnitzer, Electron and neutron electric dipole moments in the constrained MSSM. Phys. Rev. D 60, 073003 (1999). arXiv:hep-ph/9903402

10. A. Pilaftsis, Higgs-mediated electric dipole moments in the MSSM: An application to baryogenesis and Higgs searches. Nucl. Phys. B 644, 263-289 (2002). arXiv:hep-ph/0207277

11. A. Bartl, W. Majerotto, W. Porod, D. Wyler, Effect of supersymmetric phases on lepton dipole moments and rare lepton decays. Phys. Rev. D 68, 053005 (2003). arXiv:hep-ph/0306050

12. V.D. Barger et al., CP-violating phases in SUSY, electric dipole moments, and linear colliders. Phys. Rev. D 64, 056007 (2001). arXiv:hep-ph/0101106

13. M. Pospelov, A. Ritz, Electric dipole moments as probes of new. Ann. Phys. 318, 119-169 (2005). arXiv:hep-ph/0504231

14. K.A. Olive, M. Pospelov, A. Ritz, Y. Santoso, CP-odd phase correlations and electric dipole moments. Phys. Rev. D 72, 075001 (2005). arXiv:hep-ph/0506106

15. S. Abel, O. Lebedev, Neutron electron EDM correlations in supersymmetry and prospects for EDM searches. J. High Energy Phys. 01, 133 (2006). arXiv:hep-ph/0508135

16. S. Yaser Ayazi, Y. Farzan, Reconciling large CP-violating phases with bounds on the electric dipole moments in the MSSM. Phys. Rev. D 74, 055008 (2006). arXiv:hep-ph/0605272

17. J. Ellis, J.S. Lee, A. Pilaftsis, Electric dipole moments in the MSSM reloaded. arXiv:0808.1819 [hep-ph]

18. S. Kraml, CP violation in SUSY. arXiv:0710.5117 [hep-ph]

19. A. Bartl, T. Kernreiter, W. Porod, A CP sensitive asymmetry in the three-body decay stau (1) $\rightarrow b$ sneutrino/tau tau+. Phys. Lett. B 538, 59-65 (2002). arXiv:hep-ph/0202198

20. A. Bartl, H. Fraas, T. Kernreiter, O. Kittel, T-odd correlations in the decay of scalar fermions. Eur. Phys. J. C 33, 433-442 (2004). arXiv:hep-ph/0306304

21. A. Bartl, E. Christova, K. Hohenwarter-Sodek, T. Kernreiter, Triple product correlations in top squark decays. Phys. Rev. D 70, 095007 (2004). arXiv:hep-ph/0409060

22. A. Bartl, E. Christova, K. Hohenwarter-Sodek, T. Kernreiter, CP asymmetries in scalar bottom quark decays. J. High Energy Phys. 11, 076 (2006). arXiv:hep-ph/0610234

23. K. Kiers, A. Szynkman, D. London, CP violation in supersymmetric theories: stop(2) $\rightarrow$ stop(1) tau- tau+. Phys. Rev. D 74, 035004 (2006). arXiv:hep-ph/0605123
24. A. Bartl, K. Hidaka, T. Kernreiter, W. Porod, Impact of CP phases on the search for sleptons stau and sneutrino/tau. Phys. Lett. B 538, 137-145 (2002). arXiv:hep-ph/0204071

25. A. Bartl, K. Hidaka, T. Kernreiter, W. Porod, tau-sleptons and tausneutrino in the MSSM with complex parameters. Phys. Rev. D 66, 115009 (2002). arXiv:hep-ph/0207186

26. A. Bartl, S. Hesselbach, K. Hidaka, T. Kernreiter, W. Porod, Impact of CP phases on stop and sbottom searches. Phys. Lett. B 573, 153-161 (2003). arXiv:hep-ph/0307317

27. A. Bartl, S. Hesselbach, K. Hidaka, T. Kernreiter, W. Porod, Impact of $\mathrm{CP}$ phases on the search for top and bottom squarks. arXiv:hep-ph/0409347

28. A. Bartl, S. Hesselbach, K. Hidaka, T. Kernreiter, W. Porod, Top squarks and bottom squarks in the MSSM with complex parameters. Phys. Rev. D 70, 035003 (2004). arXiv:hep-ph/0311338

29. Y. Kizukuri, N. Oshimo, T odd asymmetry mediated by neutralino in $\mathrm{e}^{+} \mathrm{e}^{-}$annihilation. Phys. Lett. B 249, 449-454 (1990)

30. S.Y. Choi, H.S. Song, W.Y. Song, CP phases in correlated production and decay of neutralinos in the minimal supersymmetric standard model. Phys. Rev. D 61, 075004 (2000). arXiv:hep-ph/9907474

31. S.Y. Choi, M. Drees, B. Gaissmaier, Systematic study of the impact of CP-violating phases of the MSSM on leptonic high-energy observables. Phys. Rev. D 70, 014010 (2004). arXiv:hep-ph/0403054

32. S.Y. Choi, B.C. Chung, J. Kalinowski, Y.G. Kim, K. Rolbiecki, Analysis of the neutralino system in three-body leptonic decays of neutralinos. Eur. Phys. J. C 46, 511-520 (2006). arXiv:hep-ph/0504122

33. T. Gajdosik, R.M. Godbole, S. Kraml, Fermion polarization in sfermion decays as a probe of CP phases in the MSSM. J. High Energy Phys. 09, 051 (2004). arXiv:hep-ph/0405167

34. T. Ibrahim, P. Nath, Effective Lagrangian for anti-q squark'(i) chi(j)+, anti-q squark'(i) chi(j)0 interactions and fermionic decays of the squarks. Phys. Rev. D 71, 055007 (2005). arXiv:hep-ph/0411272

35. C.A. Baker et al., An improved experimental limit on the electric dipole moment of the neutron. Phys. Rev. Lett. 97, 131801 (2006). arXiv:hep-ex/0602020

36. M.V. Romalis, W.C. Griffith, E.N. Fortson, A new limit on the permanent electric dipole moment of Hg-199. Phys. Rev. Lett. 86, 2505-2508 (2001). arXiv:hep-ex/0012001

37. B.C. Regan, E.D. Commins, C.J. Schmidt, D. DeMille, New limit on the electron electric dipole moment. Phys. Rev. Lett. 88, 071805 (2002)

38. A. Bartl, H. Fraas, S. Hesselbach, K. Hohenwarter-Sodek, G.A. Moortgat-Pick, A T-odd asymmetry in neutralino production and decay. J. High Energy Phys. 08, 038 (2004). arXiv:hep-ph/0406190

39. G.A. Moortgat-Pick, H. Fraas, A. Bartl, W. Majerotto, Polarization and spin effects in neutralino production and decay. Eur. Phys. J. C 9, 521-534 (1999). arXiv:hep-ph/9903220

40. H.E. Haber, Spin formalism and applications to new physics searches. arXiv:hep-ph/9405376

41. B.C. Allanach et al., The Snowmass points and slopes: Benchmarks for SUSY searches. arXiv:hep-ph/0202233

42. C. Amsler et al. (Particle Data Group Collaboration), Review of particle physics. Phys. Lett. B 667, 1 (2008)

43. A.H. Hoang, T. Teubner, Top quark pair production close to threshold: Top mass, width and momentum distribution. Phys. Rev. D 60, 114027 (1999). arXiv:hep-ph/9904468

44. W. Porod, SPheno, a program for calculating supersymmetric spectra, SUSY particle decays and SUSY particle production at $\mathrm{e}^{+} \mathrm{e}^{-}$colliders. Comput. Phys. Commun. 153, 275-315 (2003). arXiv:hep-ph/0301101

45. G.P. Lepage, A new algorithm for adaptive multidimensional integration. J. Comput. Phys. 27, 192 (1978) 
46. G.P. Lepage, VEGAS: An adaptive multidimensional integration program. Cornell preprint CLNS-80/447 (1980)

47. M. Bahr et al., Herwig++ physics and manual. arXiv:0803.0883 [hep-ph]

48. M. Bahr et al., Herwig++ 2.2 release note. arXiv:0804.3053 [hep$\mathrm{ph}]$

49. M. Gigg, P. Richardson, Simulation of beyond standard model physics in Herwig++. Eur. Phys. J. C 51, 989-1008 (2007). arXiv:hep-ph/0703199

50. R. Kleiss et al., CERN 89-08, vol. 3, pp. 129-131

51. A. Bartl, S. Hesselbach, K. Hidaka, T. Kernreiter, W. Porod, Impact of SUSY CP phases on stop and sbottom decays in the MSSM. arXiv:hep-ph/0306281
52. A.D. Martin, W.J. Stirling, R.S. Thorne, G. Watt, Update of parton distributions at NNLO. Phys. Lett. B 652, 292-299 (2007). arXiv:0706.0459 [hep-ph]

53. K. Desch, J. Kalinowski, G. Moortgat-Pick, K. Rolbiecki, W.J. Stirling, Combined LHC/ILC analysis of a SUSY scenario with heavy sfermions. J. High Energy Phys. 12, 007 (2006). arXiv:hep-ph/0607104

54. G. Weiglein et al. (LHC/LC Study Group Collaboration), Physics interplay of the LHC and the ILC. Phys. Rep. 426, 47-358 (2006). arXiv:hep-ph/0410364

55. A. Bartl, H. Fraas, W. Majerotto, Production and decay of neutralinos in $\mathrm{e}^{+} \mathrm{e}^{-}$annihilation. Nucl. Phys. B 278, 1 (1986) 